\title{
The Solidarity Value as a Probabilistic Solidarity Value
}

\author{
Serge B. Nlénd Oum, Lawrence Diffo Lambo \\ University of Yaounde I, Yaounde, Cameroon \\ Email: dydon10@yahoo.fr, yldiffo@yahoo.fr
}

How to cite this paper: Nlénd Oum, S. B., \& Diffo Lambo, L. (2020). The Solidarity Value as a Probabilistic Solidarity Value. Theoretical Economics Letters, 10, 13971425.

https://doi.org/10.4236/tel.2020.106085

Received: October 18, 2020

Accepted: December 28, 2020

Published: December 31, 2020

Copyright $\odot 2020$ by author(s) and Scientific Research Publishing Inc. This work is licensed under the Creative Commons Attribution International License (CC BY 4.0).

http://creativecommons.org/licenses/by/4.0/

(c) (i) Open Access

\begin{abstract}
Our paper focuses on two evolutions in the Shapley value: on the one hand, Radzik and Nowak's solidarity value (in short s-value) allows the needy to receive solidarity help from privileged players; on the other hand, Weber's concept of probabilistic value provides a setting where the probability of joining a coalition depends both on the player being picked to join and on the coalition being constituted, unlike for the Shapley value which assumes equal chance to all players still waiting aside. In a context where the solidarity of Nowak and Radzik is the guiding policy for profit sharing, what is the meaning of this Weber's idea of probabilistic value? We come out with a large class of values, called Probabilistic solidarity values ( $p s$-values), that comprises the $s$-value. Our two main results are a characterization of Radzik-Nowak's solidarity value as a specific Probabilistic solidarity value and a characterization of symmetric ps-values which exhibit a rational intuitive property found in literature on the per-capita productivity.
\end{abstract}

\section{Keywords}

Game Theory, Shapley Value, Solidarity Value, Probabilistic Value, Probabilistic System

\section{Introduction}

Value theory started with the Shapley value defined by Shapley (1953). The bargaining model of the Shapley value is based on two main assumptions: a player who joins a coalition gets the whole of his marginal contribution, and all players enjoy equal chance of joining any given coalition being constituted. The solidarity value presented by Nowak and Radzik (1994) agrees with the Shapley value about the principle of equal opportunity of admission into any given coalition, but instead distributes the player's marginal contribution in equal shares to the 
newcomer and all others admitted earlier (since every coalition can be formed and each player receives the average marginal contribution of a member of the formed coalition). The probabilistic values of Weber (1988) are a generalization of the Shapley value where the newcomer keeps the whole of his marginal contribution, and where coalition formation is modelled by assuming that every player is endowed with an a priori personal probability distribution over the set of all coalitions not containing him, which quantifies the chance of that player getting admitted. Hence, the chance of being admitted into a coalition depends both on that coalition and on the player being considered for admission.

Note that with probabilistic values, Weber challenges the principle of equal chance in joining any coalition by assuming that two different players can have different views about joining that coalition. In the present work, we extend this approach to the solidarity of Nowak and Radzik. And we need to understand how that solidarity works in a context where players can individually assess their positions in the game.

Following this introduction, Section 2 recalls some preliminaries for values and states the definition of probabilistic solidarity values (ps-values). In Section 3 , we characterize symmetric ps-values among the collection of all ps-values. Section 4 introduces the $A$-consistent player axiom and the solidarity-monotony axiom, and following Weber (1988), we study the impact on a value of axioms of linearity, solidarity-monotony and A-consistent player property. And we obtain an axiomatization of the whole class of ps-values. In Section 5, we characterize efficient probabilistic values and we identify the solidarity value in the class of probabilistic solidarity values. An intuitive property for solidarity solutions, found in literature, based on the per-capita productivity is examined in Section 6, where we characterize symmetric ps-values which satisfy this property. The conclusion and some outlooks end the paper.

\section{Definitions and Notations}

We fix a particular set $N=\{1,2, \cdots, n\}$ of elements called the players. The collection of coalitions (or subsets) of $N$ is denoted by $P(N)$. A TU game on $N$ is a real-valued function $v: P(N) \rightarrow \mathbb{R}$ that assigns "a worth" to each coalition and satisfies $v(\varnothing)=0$. Let $\Gamma$ be the collection of all TU games on $N$ (note that $\Gamma$ is a $2^{n}$-dimensional vector space). The game $v$ is monotonous when $\forall(S, T) \subseteq N \times N, \quad[T \subseteq S] \Rightarrow[v(S) \geq v(T)]$. A value on $\Gamma$ is a function $f: \Gamma \rightarrow \mathbb{R}^{n}$, and for every $v \in \Gamma, f(v)$ is denoted by $f(v)=\left(f_{i}(v)\right)_{i \in N}$. For each player $i, f_{i}(v)$ measures the material gain (or a quantity of wealth) that he receives as a result of playing that game. Let us recall some of the properties usually investigated about a value $f$.

Axiom 1 (Linearity) The linearity axiom holds when $\forall\left(v_{1}, v_{2}\right) \in \Gamma \times \Gamma, \forall c \in \mathbb{R}, \quad f\left(c v_{1}+v_{2}\right)=c f\left(v_{1}\right)+f\left(v_{2}\right)$.

(Additivity) The additivity axiom is satisfied when $\forall\left(v_{1}, v_{2}\right) \in \Gamma \times \Gamma$, $f\left(v_{1}+v_{2}\right)=f\left(v_{1}\right)+f\left(v_{2}\right)$ 
(Monotony) Monotony holds for $f$ when for every player $i$ and for every monotonous game $v, \quad f_{i}(v) \geq 0$.

(Efficiency) A value $f$ satisfies the efficiency axiom when $\forall v \in \Gamma, \forall i \in N$, $\sum_{i \in N} f_{i}(v)=v(N)$.

(Symmetry) Symmetry is satisfied when for each permutation on $N$ denoted by $\theta$, we have for every game $v, f_{\theta(i)}(v)=f_{i}(\theta v)$ (where $\theta v$ is the TU game on $N$ defined by $\forall S \subseteq N, \theta v(S)=v(\theta(S)))$.

(The null player) A value $f$ satisfies the null player axiom when for every $v \in \Gamma$ and every $i \in N$ such that $i$ is null in the game $v$ (that is $v(T)-v(T \backslash\{i\})=0$ for every coalition $T$ containing $\hat{i})$, we have $f_{i}(v)=0$.

(The $A$-null player) A value $f$ satisfies the $A$-null player axiom when for every $v \in \Gamma$ and every $i \in N$ such that $i$ is $A$-null in the game $v$ (that is $A^{v}(T)=\frac{1}{|T|} \sum_{k \in T}[v(T)-v(T \backslash k)]=0$ for every coalition $T$ containing $\left.i\right)$, we have $f_{i}(v)=0$.

For short, we often omit braces and write $i$ for $\{i\}$. An example of value is given by the Shapley value in Shapley (1953), and the Shapley value of players for playing the game $v \in \Gamma$ is defined by:

$$
\forall i \in N, \operatorname{Shap}_{i}(v)=\sum_{T / i \in T} \frac{(n-|T|) !|| T|-1| !}{n !}[v(T)-v(T \backslash i)]
$$

In fact $\operatorname{Shap}_{i}(v)$ can be interpreted as the expected marginal contribution of player $i$ for playing the game $v$, when the coalition formation policy is that all players assemble in the grand coalition $N$ by a one by one admission, each in turn. At the stage where some coalition not containing $i$ is constituted, needs $i$ as the next newcomer to be completed into $T$ in the way of growing to lastly become $N$, it is assumed equality of chance to all the remaining players including $i$ to be picked for admission. That chance for picking $i$ is therefore $\frac{1}{n-|T|+1}$ in a specific occurrence, but amounts to $\frac{(n-|T|) !(|T|-1) !}{n !}$ when we take into account all the possible occurrences where a coalition not containing $i$ needs the newcomer $i$ to be completed into $T$. As for the profit sharing policy, it is assumed that upon joining $T$, the player $i$ gets (and keeps for himself) his marginal contribution which is $v(T \cup i)-v(T)$. The Shapley value was characterized by Shapley (1953) as the unique value satisfying the efficiency, the additivity, the symmetry and the null player axioms. In the probabilistic values Weber (1988) defines for each player $i$ a probability distribution over the collection of all coalitions not containing him as a function $p^{i}: P(N \backslash i) \rightarrow R$, such that $\forall T \subseteq N \backslash\{i\}, \quad p^{i}(T) \geq 0$ and $\sum_{T \subseteq N \backslash i} p^{i}(T)=1$. The real number $p^{i}(T)$, denoted by $p^{i}(T)=p_{T}^{i}$, is interpreted as the (subjective) probability that player $i$ joins coalition $T$ at the stage where $T$ is constituted en route to grow and become 
the grand coalition $N$.

For every $p=\left(\left(p_{T}^{i}\right)_{T \subset N \backslash i}\right)_{i \in N}$, the probabilistic value $\tilde{f}=\left(\tilde{f}_{i}(v)\right)_{i=1,2, \cdots, n}$ of players for playing the game $v \in \Gamma$ is defined by:

$$
\forall i \in N, \quad \tilde{f}_{i}(v)=\sum_{T \subset N \backslash i} p_{T}^{i}[v(T \bigcup i)-v(T)]
$$

In this paper, any family of probability distribution $p=\left(p^{i}\right)_{i \in N}$, also denoted by $p=\left(\left(p_{T}^{i}\right)_{T \subset N \backslash i}\right)_{i \in N}$ is called a probability system. If a value $\tilde{f}=\left(\tilde{f}_{i}(v)\right)_{i=1,2, \cdots, n}$ is defined by $\forall i \in N, \quad \tilde{f}_{i}(v)=\sum_{T \subset N \backslash i} p_{T}^{i}[v(T \cup i)-v(T)]$, then $p$ is called the probability system associated with $\tilde{f}$. So for any player $i \in N, \tilde{f}_{i}(v)$ can be interpreted as the expected marginal contribution of player $i$ for playing the game $v$ when it is not assumed that two different players have equal chance of joining some coalition. It is clear that probabilistic values generalize the Shapley value. For the Shapley value, $p_{T \backslash i}^{i}=\frac{(n-|T|) !(|T|-1) !}{n !}$ when $i \in T$, and $p_{T}^{i}=\frac{(n-|T|-1) !(|T|) !}{n !}$, when $i \notin T$.

The Shapley value is characterized by Weber (1988) as the unique probabilistic value satisfying both the efficiency and symmetry axioms. The solidarity value of Radzik (1994) is introduced to express some idea of solidarity among players. Indeed for each player $i \in N$ and for every coalition $T$ containing $i$, Nowak and Radzik express this solidarity by replacing, in the Shapley value formula, the marginal contribution $v(T)-v(T \backslash i)$ of player $i$ being admitted to join $T \backslash i$ with the so called average marginal contribution defined by $A^{v}(T)=\frac{1}{|T|} \sum_{k \in T}[v(T)-v(T \backslash k)]$. The solidarity value $\varphi=\left(\varphi_{i}\right)_{i=1,2, \cdots, n}$ of players for playing the game $v$ is defined by:

$$
\forall i \in N, \varphi_{i}(v)=\sum_{T / i \in T} \frac{(n-|T|) !(|T|-1) !}{n !} A^{v}(T) .
$$

It comes out that in the solidarity value, each player instead of carrying away for himself his marginal contribution, the solidarity approach consists of collecting all marginal contributions and then distributing the total amount in equal shares to all members of $T$. Let us recall the Nowak-Radzik's characterization of the solidarity value.

Theorem 1 Nowak and Radzik (1994)

A value $f$ on $\Gamma$ is the solidarity value, if and only if, $f$ satisfies the additivity, the symmetry, the efficiency and the $A$-null player axioms.

While keeping with Nowak and Radzik's model of solidarity, we want to implement Weber's idea about a player $i$ joining a coalition $T \backslash\{i\}$ by chance following an a priori probability $P_{T \backslash i}^{i}$.

If we assume equality of chance at every admission of players to those yet to 
join, then the probability that during the building up of the grand coalition (which is possible in $|N|$ ! ways of equal probability $\frac{1}{|N| !}$ ), player $i$ is admitted to join only when the set of players already recruited is $T \backslash i$ is $\frac{(|T|-1) !(|N|-|T|) !}{|N| !}$. So, Shapley's formula is a particular case of Weber's with $P_{T \backslash i}^{i}$ expressed as follows:

$$
P_{T \backslash i}^{i}=\frac{(|T|-1) !(|N|-|T|) !}{|N| !}
$$

Weber's formula for probabilistic values reads $\tilde{f}_{i}(v)=\sum_{T / i \in T} p_{T \backslash i}^{i}[v(T)-v(T \backslash i)]$. So, we obtain the solidarity of Nowak and Radzik from the probabilistic value by substituting $[v(T)-v(T \backslash i)]$ for $A^{v}(T)$. And we can define the more general concept of probabilistic solidarity value as follows:

Definition 1 Given a probability system $p=\left(\left(p_{T}^{i}\right)_{T \subseteq N \backslash i}\right)_{i \in N}$, the probabilistic solidarity value associated with $p$ is the value $\phi$ defined by $\forall v \in \Gamma$, $\forall i \in N$

$$
\phi_{i}(v)=\sum_{T / i \notin T} p_{T}^{i} A^{v}(T \bigcup i)
$$

From now on, instead of "probabilistic solidarity value", we shortly write "ps-value".

Following Weber, we say that player $i$ views his participation in a game as joining some coalition $T$ and receiving as reward the average marginal contributions $A^{v}(T \cup i)$ with a (subjective) probability $p_{T}^{i}$ that he joins $T$. So $\phi_{i}(v)$ is the expected payoff of $i$ from the game.

It is clear that, the solidarity value is a particular ps-value with the probability system $p$ defined by:

$$
p_{T}^{i}=\frac{(n-|T|-1) !(|T|) !}{n !} .
$$

In the following section, we characterize symmetric ps-values among the class of all ps-values. Our result recalls the one obtained in Weber (1988) for probabilistic values.

\section{Symmetric ps-Values}

By proposition 2 below, we claim that when a ps-value is symmetric, then the probability of joining some coalition only depends on the cardinality of that coalition.

Proposition 2 If a ps-value $\phi$ on $\Gamma$ with a probability system $p$ satisfies the symmetryaxiom, then for every $j \in N, i \in N, T \subseteq N \backslash j$, and $S \subseteq N \backslash i$, 


$$
(|S|=|T|) \Rightarrow\left(p_{T}^{j}=p_{S}^{i}\right)
$$

Proof. For any $i \in N$, let $T_{1}$ and $T_{2}$ be two coalitions with $i \notin T_{1} \cup T_{2}$ such that $0<\left|T_{1}\right|=\left|T_{2}\right|<n-1$. Consider a permutation $\theta$ on $N$ such that $\theta\left(T_{2}\right)=T_{1}$ with $\theta(i)=i$. Consider the game $v_{T_{1}}$ defined by:

$$
v_{T_{1}}(S)=\left\{\begin{array}{cc}
0 & \text { if } S=T_{1} \text { or } S=\varnothing \\
1 & \text { otherwise }
\end{array}\right.
$$

We have $\phi_{i}\left(v_{T_{1}}\right)=\sum_{T / i \in T} p_{T \backslash i}^{i} \frac{1}{|T|}\left(\sum_{k \in T}\left[v_{T_{1}}(T)-v_{T_{1}}(T \backslash k)\right]\right)$,

But with $i \in T$ we always have $v_{T_{1}}(T)=1$, so $v_{T_{1}}(T)-v_{T_{1}}(T \backslash j) \neq 0$ implies that $v_{T_{1}}(T \backslash j)=0$, i.e. $T \backslash j=T_{1}$ or $T \backslash j=\varnothing$. But $T \backslash j=\varnothing$ means $T=\{i\}$ and $j=i$, while $T \backslash j=T_{1}$ means $j=i$ and $T=T_{1} \cup\{i\}$. Therefore,

$$
\begin{aligned}
\phi_{i}\left(v_{T_{1}}\right)= & {\left[p_{T \backslash i}^{i} \frac{1}{|T|}\left[v_{T_{1}}(T)-v_{T_{1}}(T \backslash j)\right]\right]_{T=\{i\}, j=i} } \\
& +\left[p_{T \backslash i}^{i} \frac{1}{|T|}\left[v_{T_{1}}(T)-v_{T_{1}}(T \backslash j)\right]\right]_{T=T_{1} \cup\{i\}, j=i} \\
= & p_{\varnothing}^{i} \frac{1}{1}\left[1-v_{T_{1}}(\varnothing)\right]+p_{T_{1}}^{i} \frac{1}{\left|T_{1}\right|+1}\left[1-v_{T_{1}}\left(T_{1}\right)\right] \\
= & p_{\varnothing}^{i}+p_{T_{1}}^{i} \frac{1}{\left|T_{1}\right|+1}
\end{aligned}
$$

Furthermore $\theta v_{T_{1}}=v_{T_{2}}$ and $\phi_{i}\left(v_{T_{2}}\right)=p_{\varnothing}^{i}+p_{T_{2}}^{i} \times \frac{1}{\left|T_{2}\right|+1}$. By symmetry axiom, we have $\phi_{i}\left(v_{T_{1}}\right)=\phi_{i}\left(v_{T_{2}}\right)$.

Thus $p_{T_{1}}^{i}=p_{T_{2}}^{i}$ (i).

Next for distinct players $i$ and $j$, let $\theta$ be a permutation on $N$ that interchanges $i$ and $j$, while leaving the remaining players fixed. Consider the game $v$ defined by:

$$
v(S)=\left\{\begin{array}{lc}
1 & \text { if } S \neq \varnothing \\
0 & \text { else }
\end{array}\right.
$$

We obtain $\forall k \in N, \phi_{k}(v)=p_{\varnothing}^{k}$. Note that $\theta v=v$. That implies (by symmetry axiom ) $\phi_{i}(v)=\phi_{j}(v)$. Hence $p_{\varnothing}^{i}=p_{\varnothing}^{j}$ (ii).

Again for distinct players $i$ and $j$, let $\theta$ be a permutation that transposes $i$ and $j$, while leaving the remaining players fixed. Let $T_{0} \subseteq N \backslash\{i, j\}$ be a non empty coalition and consider the game $v_{T_{0}}$ defined by:

$$
v_{T_{0}}(S)=\left\{\begin{array}{cc}
0 & \text { if } S=T_{0} \text { or } S=\varnothing \\
1 & \text { else }
\end{array}\right.
$$

We obtain $\phi_{i}\left(v_{T_{0}}\right)=p_{\varnothing}^{i}+p_{T_{0}}^{i} \times \frac{1}{\left|T_{0}\right|+1}$. Note that $\theta v_{T_{0}}=v_{T_{0}}$, and by the 
symmetry axiom we have $\phi_{i}\left(v_{T_{0}}\right)=\phi_{j}\left(v_{T_{0}}\right)=p_{\varnothing}^{j}+p_{T_{0}}^{j} \times \frac{1}{\left|T_{0}\right|+1}$.

So $p_{\varnothing}^{i}+p_{T_{0}}^{i} \times \frac{1}{\left|T_{0}\right|+1}=p_{\varnothing}^{j}+p_{T_{0}}^{j} \times \frac{1}{\left|T_{0}\right|+1}$. Using (ii), we obtain $p_{T_{0}}^{i}=p_{T_{0}}^{j}$ (iii).

For distinct players $i$ and $j$, let $\theta$ be a permutation that interchanges $i$ and $j$, while leaving the remaining players fixed. Let $T_{3} \subseteq N \backslash j, i \in T_{3}$ and consider the game $v_{T_{3}}$ defined by:

$$
v_{T_{3}}(S)=\left\{\begin{array}{cc}
0 & \text { if } S=T_{3} \text { or } S=\varnothing \\
1 & \text { else }
\end{array}\right.
$$

We have $\phi_{j}\left(v_{T_{3}}\right)=p_{\varnothing}^{j}+p_{T_{3}}^{j} \times \frac{1}{\left|T_{3}\right|+1}$. Note that $\theta v_{T_{3}}=v_{\tilde{T}_{3}}$ with

$\tilde{T}_{3}=\left(T_{3} \backslash i\right) \cup j$. By symmetry axiom we have

$\phi_{j}\left(v_{T_{3}}\right)=\phi_{i}\left(v_{\tilde{T}_{3}}\right)=p_{\varnothing}^{i}+p_{\tilde{T}_{3}}^{i} \times \frac{1}{\left|\tilde{T}_{3}\right|+1}$.

Using (ii), we have $p_{T_{3}}^{j}=p_{\tilde{T}_{3}}^{i}$ (iv).

Once more, for distinct players $i$ and $j$, let $\theta$ be a permutation that interchanges $i$ and $j$, while leaving the remaining players fixed. Consider the game $\hat{v}^{i}$ defined by

$$
\hat{v}^{i}(S)=\left\{\begin{array}{lc}
|S|+1 & \text { if } i \notin S, S \neq \varnothing \\
0 & \text { otherwise }
\end{array}\right.
$$

We have $\phi_{i}\left(\hat{v}^{i}\right)=-\sum_{T / i \in T} p_{T \backslash i}^{i}$. Since $\pi \hat{v}^{i}=\hat{v}^{j}$. By symmetry axiom, we have $\phi_{i}\left(\hat{v}^{i}\right)=\phi_{j}\left(\hat{v}^{j}\right)=-\sum_{T / j \in T} p_{T \backslash j}^{j}$ i.e. $\sum_{T / i \in T} p_{T \backslash i}^{i}=\sum_{T / j \in T} p_{T \backslash j}^{j}$.

So, $p_{N \backslash i}^{i}+\sum_{T / T \neq N, i \in T} p_{T \backslash i}^{i}=p_{N \backslash j}^{j}+\sum_{T / T \neq N, j \in T} p_{T \backslash j}^{j}$. Using (ii), (iii) and (iv), we obtain $p_{N \backslash i}^{i}=p_{N \backslash j}^{j}(\mathrm{v})$.

Finally with (i), (ii), (iii), (iv) and (v), we have the proof.

Now we show that the converse of proposition 2 holds.

Proposition 3 Let $\phi$ be a ps-value on $\Gamma$ with a probability system $p$. If for every $j \in N, i \in N, T \subseteq N \backslash j$ and $S \subseteq N \backslash i,(|S|=|T|) \Rightarrow\left(p_{T}^{j}=p_{S}^{i}\right)$, then $\phi$ satisfies the symmetry axiom.

Proof. We have $\forall i \in N, \phi_{i}(v)=\sum_{T / i \in T} p_{T \backslash i}^{i} A^{v}(T)$. Consider $\theta$, a permutation on $N$. We should prove that for any $i \in N, \phi_{\theta(i)}(v)=\phi_{i}(\theta v)$. We have

$$
\begin{aligned}
& \phi_{i}(\theta v)=\sum_{T / i \in T} p_{T \backslash i}^{i} \frac{1}{|T|} \sum_{k \in T}[\theta v(T)-\theta v(T \backslash k)] \\
& \phi_{\theta(i)}(v)=\sum_{T / \theta(i) \in T} p_{T \backslash \theta(i)}^{\theta(i)} \frac{1}{|T|} \sum_{k \in T}[v(T)-v(T \backslash k)]
\end{aligned}
$$

To prove that equality, denote by $A=\{T \subseteq N / i \in T\}$ and 
$B=\{T \subseteq N / \theta(i) \in T\}$. Remark that $\theta: j \mapsto j$ is a bijection from $N$ to $N$, so $\theta: T \mapsto \theta(T)$, seen as an application from $P(N)$ to $P(N)$ is also a bijection. Also, it is straightforward that $\forall T \in A, \quad \theta(T) \in B$, while $\forall T \in B$, $\theta^{-1}(T) \in A$. Therefore, $\theta$, seen as an application from $A$ to $B$ is also a bijection. Let us first prove that for every $T \in A, \hat{T}=\theta(T)$ satisfies (a) below:

$$
p_{T \backslash i}^{i} \frac{1}{|T|} \sum_{k \in T}[\theta v(T)-\theta v(T \backslash k)]=p_{\hat{T} \backslash \theta(i)}^{\theta(i)} \frac{1}{|\hat{T}|} \sum_{k \in \hat{T}}[v(\hat{T})-v(\hat{T} \backslash k)]
$$

Indeed, $p_{T \backslash i}^{i} \frac{1}{|T|}=p_{T \backslash \theta(i)}^{\theta(i)} \frac{1}{|\hat{T}|}$, due to the fact that $|\hat{T}|=|T|$ and

$(|S|=|T|) \Rightarrow\left(p_{T}^{j}=p_{S}^{i}\right)$. We need to show that $(\mathrm{b})$ holds:

$$
\sum_{k \in T}[\theta v(T)-\theta v(T \backslash k)]=\sum_{k \in \hat{T}}[v(\hat{T})-v(\hat{T} \backslash k)]
$$

But $\theta: k \mapsto \theta(k)$, seen as an application from $T$ to $\hat{T}$ is also a bijection, and for any $k \in T, \hat{k}=\theta(k)$ belongs to $\hat{T}$ and satisfies

$$
v(\hat{T})-v(\hat{T} \backslash \hat{k})=\theta v(T)-\theta v(T \backslash k)
$$

To check for (c),

$$
\begin{aligned}
v(\hat{T})-v(\hat{T} \backslash \hat{k}) & =v(\theta(T))-v(\theta(T) \backslash \theta(k)) \\
& =v(\theta(T))-v(\theta(T \backslash k)) \\
& =\theta v(T)-\theta v(T \backslash k)
\end{aligned}
$$

So, (c) is true, which leads to (b) being true, and therefore (a) is true. So, $\phi_{\theta(i)}(v)=\phi_{i}(\theta v)$.

The two propositions 2 and 3 lead to the following result:

Theorem 4 A ps-value $\phi$ on $\Gamma$ with a probability system $p$ satisfies the symmetry axiom, if and only if for every $j \in N, i \in N, T \subseteq N \backslash j$, and $S \subseteq N \backslash i,(|S|=|T|) \Rightarrow\left(p_{T}^{j}=p_{S}^{i}\right)$.

In the next section, we introduce the axioms of A-consistent player and s-monotony. And we study their impacts on a value.

\section{About the Linearity, the Solidarity Monotony and the A-Consistent Player Axioms for a Value}

The A-null axiom in Nowak and Radzik (1994) ensures that each A-null player receives a 0 payoff. And what about a player for which the average marginal contribution of each coalition containing him is a fixed non null real number? One can understand that the A-null player concept can be generalized by considering any $c \in \mathbb{R}$ which is not necessarily equal to 0 . And we state that a player $i$ is $A$-consistent at $c$ for $v \in \Gamma$ when $\forall T \subseteq N, i \in T \Rightarrow A^{v}(T)=c$. So, being A-null is the same as being A-consistent at 0 . It is easy to verify that if two different players are respectively A-consistent at $c_{1} \in \mathbb{R}$ and $c_{2} \in \mathbb{R}$ for $v \in \Gamma$, then we have $c_{1}=c_{2}$. Indeed, we have $A^{v}(\{i, j\})=c_{1}$ and $A^{v}(\{i, j\})=c_{2}$. One may wonder if it is possible to be an A-consistent player without being a null 
player. A glance at additive games provides an affirmative answer. Indeed, it is easy to prove that if a game $v$ is additive, then there exists an A-consistent player if and only if all players are A-consistent, and this happens if and only if $\forall i \in N$, $\forall j \in N \quad v(i)=v(j)=c$. So, for the additive game satisfying $\forall i \in N, v(i)=3$, all players are A-consistent at 3.

We introduce the A-consistent player axiom as follows:

Axiom $2 A$ value $f$ on $\Gamma$ satisfies the $A$-consistent player axiom when for every $v \in \Gamma$, for every player $i$ and every $c \in \mathbb{R}$ such that $i$ is $A$-consistent at $c$ for $v$, we have $f_{i}(v)=c$.

We claim that if each coalition containing player $i$ has a null or not average marginal contribution $c$, then according to the A-consistent player axiom, player $i$ receives $c$ payoff from the game.

Note that the Shapley value fails to satisfy the A-consistent player axiom. Indeed, for $N=\{1,2,3\}$, consider the game $v$ defined by $v(1)=\frac{1}{3}$,

$v(2)=v(3)=1$ and $v(\{1,2\})=v(\{1,3\})=v(N)=1$ and $v(\{2,3\})=0$. Player 1

is an A-consistent player at $\frac{1}{3}$ for $v$, because every $T \subseteq N$ with $1 \in T$ satisfies $A^{v}(T)=\frac{1}{3}$, but $\operatorname{Shap}_{1}(v)=\frac{4}{9}$.

Lemma 5 The solidarity value satisfies the A-consistent player axiom.

Proof. For each $v \in \Gamma$, the solidarity value is defined by: $\forall j \in N$, $\varphi_{j}(v)=\sum_{T \ni j} p_{T \backslash j}^{j} A^{v}(T)$ where

$$
p_{T \backslash j}^{j}=\frac{(n-|T|) !(|T|-1) !}{n !} \text {. Let } i \in N \text { and } c \in \mathbb{R} \text { such that } i \text { is an }
$$

A-consistent at $c$ for $v$. So, $\forall T \subseteq N, i \in T, A^{v}(T)=c$. Furthermore, the solidarity value $\varphi$ is a ps-value. So $\forall j \in N, \sum_{T / j \in T} p_{T \backslash j}^{j}=1$. Thus,

$$
\begin{aligned}
\varphi_{i}(v) & =\sum_{T / i \in T} p_{T \backslash i}^{i} A^{v}(T) \\
& =c \sum_{T / i \in T} p_{T \backslash j}^{j}, \text { since } i \in T \Rightarrow A^{v}(T)=c \\
& =c \text { since } \sum_{T / i \in T} p_{T \backslash i}^{i}=1
\end{aligned}
$$

and the solidarity value satisfies the A-consistent player axiom.

The A-consistent player axiom is new and by lemma 6 below, we easily see that it differs with Nowak and Radzik's A-null axiom.

Lemma 6 1) If a value $f$ on $\Gamma$ satisfies the $A$-consistent player axiom, then $f$ satisfies the A-null player axiom.

2) A value $f$ on $\Gamma$ can satisfy the A-null player axiom, and fail to satisfy the A-consistent player axiom.

Proof. 1 ) is obvious. For 2), take any family of real numbers

$\left(a_{T}^{i}\right)_{(i, T) / i \in N, T \subseteq N, i \in T}$ verifying $\sum_{T \ni 1} a_{T}^{1} \neq 1$, and consider the value $f$ on $\Gamma$ defined 
by $\forall i \in N, \quad f_{i}(v)=\sum_{T / T \ni i} a_{T}^{i} A^{v}(T)$. It is obvious that if $i$ is A-null in $v$, then $f_{i}(v)=0$.

But now, consider the additive game $v \in \Gamma$, such that for each player $i \in N$, $v(i)=1$. It is clear that $\forall T \subseteq N, 1 \in T, A^{v}(T)=1$ holds. And $f_{1}(v)=\sum_{T / T \ni 1} a_{T}^{1} \neq 1$.

So, we have the proof of 2).

Now consider the family of games $\left(w_{T}\right)_{T \in 2^{N}} \quad\left(\right.$ where $\left.2^{N}=\{T \subseteq N, T \neq \varnothing\}\right)$ defined by

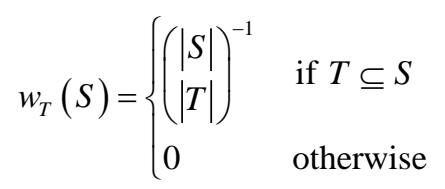

Note that the family $\left(w_{T}\right)_{T \in 2^{N}}$ is introduced in Nowak and Radzik (1994), and shown to be a vector basis for $\Gamma$. Let us recall the following result.

Lemma 7 Nowak and Radzik (1994) For any non-empty coalition $T \subseteq N$, the game $w_{T}$ has the following properties.

(i) $w_{T}(T)=1$; (ii) If $T \subseteq S$ and $T \neq S$, then $w_{T}(S)=\frac{1}{|S|} \sum_{i \in S} w_{T}(S \backslash i)$ and (iii) every player $i \in N \backslash T$ is $A$-null in the game $w_{T}$.

The proof provided by Nowak and Radzik that $\left(w_{T}\right)_{T \in 2^{N}}$ is a vector basis does not unveil the coordinates of any $v \in \Gamma$ in that basis. From proposition 8 below, we show that the coordinate of any $v \in \Gamma$ with respect to $w_{T}, T \neq \varnothing$ is $A^{v}(T)$.

Proposition 8 For every $v \in \Gamma, v=\sum_{T / T \subseteq N, T \neq \varnothing} A^{v}(T) w_{T}$

Proof. Let $v \in \Gamma$. Since $\left(w_{T}\right)_{T \in 2^{N}}$ is a basis for $\Gamma$, there exists $\left(\alpha_{T}\right)_{T \in 2^{N}}$, a family of real numbers such that $v=\sum_{\varnothing \neq T \subseteq N} \alpha_{T} w_{T}$. So, for any $T_{0} \neq \varnothing, T_{0} \subseteq N$, we have:

$$
v\left(T_{0}\right)=\sum_{\varnothing \neq T \subseteq N} \alpha_{T} w_{T}\left(T_{0}\right) \text { and } \sum_{\varnothing \neq T \subseteq N} \alpha_{T} w_{T}\left(T_{0}\right)=\sum_{T \neq \varnothing, T \subseteq T_{0}} \alpha_{T} w_{T}\left(T_{0}\right)+\alpha_{T_{0}} . \text { By }
$$
the lemma 7 , we have

$$
\begin{aligned}
v\left(T_{0}\right) & =\sum_{T \neq \varnothing, T \subseteq T_{0}} \alpha_{T} \times\left(\frac{1}{\left|T_{0}\right|} \sum_{k \in T_{0}} w_{T}\left(T_{0} \backslash k\right)\right)+\alpha_{T_{0}} \\
& =\sum_{T \neq \varnothing, T \subseteq T_{0}}\left(\frac{1}{\left|T_{0}\right|} \sum_{k \in T_{0}} \alpha_{T} w_{T}\left(T_{0} \backslash k\right)\right)+\alpha_{T_{0}} \\
& =\sum_{k \in T_{0}}\left(\frac{1}{\left|T_{0}\right|} \sum_{T \neq \varnothing, T \subseteq T_{0}} \alpha_{T} w_{T}\left(T_{0} \backslash k\right)\right)+\alpha_{T_{0}} \\
& =\sum_{k \in T_{0}}\left(\frac{1}{\left|T_{0}\right|} \sum_{T \neq \varnothing, T \subseteq T_{0} \backslash k} \alpha_{T} w_{T}\left(T_{0} \backslash k\right)\right)+\alpha_{T_{0}}, \\
& \text { since } w_{T}\left(T_{0} \backslash k\right)=0 \text { if } k \in T,
\end{aligned}
$$


Therefore, $v\left(T_{0}\right)=\left(\frac{1}{\left|T_{0}\right|} v\left(T_{0} \backslash k\right)\right)+\alpha_{T_{0}}$, because $v=\sum_{\varnothing \neq T \subseteq N} \alpha_{T} w_{T}$ and $\left[\operatorname{non}\left(T \subseteq T_{0} \backslash k\right) \Rightarrow w_{T}\left(T_{0} \backslash k\right)=0\right]$

Hence, $v\left(T_{0}\right)=\frac{1}{\left|T_{0}\right|} \sum_{k \in T_{0}} v\left(T_{0} \backslash k\right)+\alpha_{T_{0}}$, which implies that

$$
\alpha_{T_{0}}=v\left(T_{0}\right)-\frac{1}{\left|T_{0}\right|} \sum_{k \in T_{0}} v\left(T_{0} \backslash k\right)=A^{v}\left(T_{0}\right)
$$

Therefore, $\alpha_{T_{0}}=A^{v}\left(T_{0}\right)$, which shows that $\forall T \subseteq N$ with $T \neq \varnothing$, $\alpha_{T}=A^{v}(T)$ and ends the proof.

Now we investigate in the following result, the impact of the axioms of linearity and A-consistent player for a value.

Proposition 9 Let $f$ be a value on $\Gamma$ and $v \in \Gamma$. If $f$ satisfies both the linearity and the $A$-consistent player axioms, then $\forall i \in N$,

$$
\sum_{T \mid i \in T} f_{i}\left(w_{T}\right)=1 \text { and } f_{i}(v)=\sum_{T \mid i \in T} f_{i}\left(w_{T}\right) A^{v}(T) .
$$

Proof. Since $v \in \Gamma$, by proposition 8 we have $v=\sum_{T \in 2^{N}} A^{v}(T) w_{T}$. Furthermore $f$ satisfies the linearity axiom, that leads to $\forall i \in N, \quad f_{i}(v)=\sum_{T \in 2^{N}} f_{i}\left(w_{T}\right) A^{v}(T)$.

By lemma 6, we know that $f$ satisfies the A-null player axiom. Due to lemma 7 , we know that for each non-empty coalition $T$ not containing $i$, player $i$ is A-null in the game $w_{T}$. Thus $\forall T \subseteq N \backslash\{i\}, f_{i}\left(w_{T}\right)=0$.

So $\forall i \in N, \quad f_{i}(v)=\sum_{T \in 2^{N}} f_{i}\left(w_{T}\right) A^{v}(T)$ implies that $\forall i \in N$,

$$
f_{i}(v)=\sum_{T \mid i \in T} f_{i}\left(w_{T}\right) A^{v}(T) .
$$

Now consider the additive game $\tilde{v}$ defined by: for every player $j \in N$, $\tilde{v}(j)=c, c \in \mathbb{R}^{*}$.

We have for each $i \in N, \quad f_{i}(\tilde{v})=\sum_{T \mid i \in T} f_{i}\left(w_{T}\right) A^{\tilde{v}}(T)$. Note that every player $j$ is A-consistent at $c$ for $\tilde{v}$, since he verifies $\left[\forall T \subseteq N, j \in T \Rightarrow A^{\tilde{v}}(T)=c\right]$.

So, for each $i \in N, f_{i}(\tilde{v})=\sum_{T \mid i \in T} f_{i}\left(w_{T}\right) \times c$. And by the A-consistent player axiom, we have for each $i \in N, f_{i}(\tilde{v})=c$. That leads to $\forall i \in N$, $\sum_{T \mid i \in T} f_{i}\left(w_{T}\right) \times c=c$. Therefore for every $i \in N, \sum_{T \mid i \in T} f_{i}\left(w_{T}\right)=1$, and this ends the proof.

To motivate the monotony axiom, Weber (1988) points out that, player $i$ knows that his joining a coalition never hurts it collectively, since for every $T \subset N \backslash i, v(T \bigcup i)-v(T) \geq 0$. So, the monotony axiom ensures that player $i$ receives at least 0 as his ultimate payoff. However in the solidarity context of Nowak and Radzik, one can notice that for every game $v$, each player of a non empty coalition $T$ receives $A^{v}(T)$. So in such a context, should the positivity of a value not be defined with respect to all the average marginal contribution of 
non empty coalitions? That suggest us the solidarity monotony (or s-monotony) axiom, which can be viewed as a solidarity variant of the monotony axiom.

Definition 2 A game $v \in \Gamma$ is s-monotonic when for every non empty coalition $T, A^{v}(T) \geq 0$.

We claim that in a s-monotonic game, the assistance of the needy players in each non empty coalition does not create any debt for the wealthy ones. Therefore by the s-monotony axiom, every player receives a non negative payoff. It is obvious that any monotonic game $v \in \Gamma$ is s-monotonic. To see that the converse is false, check that when $T \neq N, w_{T}$ is s-monotonic, but fails to be monotonic.

Axiom $3 A$ value $f$ on $\Gamma$ satisfies the s-monotony axiom when for every player $i$ and for every s-monotonic game $v$, we have $f_{i}(v) \geq 0$.

The s-monotony axiom expresses a variant of the classic positivity in the sens of the solidarity of Nowak and Radzik.

Now let us study the s-monotony axiom for a value.

Proposition 10 Let $f$ be a value on $\Gamma$ such that $\forall v \in \Gamma$, for every player $i \in N, \quad f_{i}(v)=\sum_{T \mid i \in T} f_{i}\left(w_{T}\right) A^{v}(T)$ and $\sum_{T \mid i \in T} f_{i}\left(w_{T}\right)=1$. If $f$ satisfies the $s$-monotony axiom, then for every $i \in N,\left(f_{i}\left(w_{T}\right)\right)_{T \subseteq N, i \in T}$ is a probability distribution on the subsets of $N \backslash i$.

Proof. It will be sufficient to show that $\forall i \in N, \forall T \subseteq N$, $i \in T \Rightarrow f_{i}\left(w_{T}\right) \geq 0$. By lemma 7 , we know that for every non empty coalition $T$, the game $w_{T}$ is s-monotonic. So, by the s-monotony axiom, we have $\forall i \in N$, $\forall T \subseteq N, \quad i \in T, f_{i}\left(w_{T}\right) \geq 0$.

Due to proposition 9 and proposition 10, we have the following result which is an axiomatic characterization of the whole class of ps-values:

Theorem $11 A$ value $f$ on $\Gamma$ satisfies the linearity, the A-consistent player and the $s$-monotony axioms, if and only if, $f$ is a ps-value.

Proof. If $f$ satisfies both the linearity and the A-consistent player axioms, then by proposition 9 , we have $\forall i \in N, f_{i}(v)=\sum_{T \mid i \in T} f_{i}\left(w_{T}\right) A^{v}(T)$ and

$\sum_{T \mid i \in T} f_{i}\left(w_{T}\right)=1$. And using proposition 10, we have that for each player $i \in N$, the family $\left(f_{i}\left(w_{T}\right)\right)_{T \subseteq N, i \in T}$ is a probability distribution on the subsets of $N \backslash i$, where $\forall i \in N, \forall T \subseteq N, i \in T, f_{i}\left(w_{T}\right)=p_{T \backslash i}^{i}$, since $f$ satisfies the s-monotonic axiom.

Conversely, assume that there exists a probabilistic system $p=\left(\left(p_{T \backslash i}^{i}\right)_{T \subseteq N, i \in T}\right)_{i \in N}$, such that for every $v \in \Gamma$, and for every $i \in N$, $f_{i}(v)=\sum_{T \mid i \in T} p_{T \backslash i}^{i} A^{v}(T)$. It is easy to check that each application $v \mapsto A^{v}(T)$ from $\Gamma$ to $\mathbb{R}$ is linear, so $f$ satisfies the linearity axiom.

Furthermore, for each $v \in \Gamma$, let $i \in N$ such that $i$ is an A-consistent player for $v$ at $c \in \mathbb{R}$, then $\forall T \subseteq N, i \in T, A^{v}(T)=c$. We have $\sum_{T \mid i \in T} p_{T \backslash i}^{i}=1$, since 
$p^{i}=\left(p_{T \backslash i}^{i}\right)_{T \subseteq N, i \in T}$ is a probability distribution on the subsets of $N \backslash i$. Hence $f_{i}(v)=c$ and $f$ satisfies the A-consistent player axiom.

Now for every $v \in \Gamma$ such that $v$ is a s-monotonic game, we have $\forall i \in N$, $\forall T \subseteq N, i \in T, A^{v}(T) \geq 0$. So $\forall i \in N, f_{i}(v) \geq 0$ since $p_{T \backslash i}^{i} \geq 0$. That ends the proof.

In the next section, we characterize efficient ps-values and identify the solidarity value as a specific ps-value.

\section{Characterization of the Solidarity Value in the Class of ps-Values}

Theorem 12 below presents a characterization of efficient ps-values based on the probabilistic system $p$.

Theorem 12 A ps-value with probability system $p$ satisfies the efficiency axiom, if and only if,

$$
\begin{aligned}
& \sum_{i \in N} p_{N \backslash i}^{i}=1 \text { and } \forall T \notin\{\varnothing, N\}, \forall(i, j) \in N \times N, \\
& \sum_{i \in T} p_{T \backslash i}^{i}=\frac{1}{|T|+1} \sum_{L: L L|=T|+1, L \supseteq T} \sum_{j \in L} p_{L \backslash j}^{j} .
\end{aligned}
$$

Proof. Let $f$ be a ps-value, for each $v \in \Gamma$, there exists a probabilistic system $p=\left(\left(p_{T}^{i}\right)_{T \subseteq N \backslash i}\right)_{i \in N}$ such that $\forall i \in N, \quad f_{i}(v)=\sum_{T / i \in T} p_{T \backslash i}^{i} \frac{1}{|T|} \sum_{k \in T}[v(T)-v(T \backslash k)]$.

We have $\sum_{i \in N} f_{i}(v)=\sum_{i \in N} \sum_{T / i \in T} p_{T \backslash i}^{i} \frac{1}{|T|} \sum_{k \in T}[v(T)-v(T \backslash k)]$. Explicitely, we obtain:

$$
\sum_{i \in N} f_{i}(v)=\sum_{i \in N} \sum_{t=0}^{n-1} \sum_{T: T T \mid=t, i \notin T} p_{T}^{i} \frac{1}{|T|+1} \sum_{k \in T \cup i}[v(T \bigcup i)-v((T \bigcup i) \backslash k)]
$$

And

$$
\sum_{i \in N} f_{i}(v)=\sum_{T \neq \varnothing, T \neq N} v(T)\left[\sum_{i \in T} p_{T \backslash i}^{i}-\frac{1}{|T|+1} \sum_{L:|L|=T \mid+1, L \supseteq T} \sum_{j \in L} p_{L \backslash j}^{j}\right]+v(N) \sum_{i \in N} p_{N \backslash i}^{i}
$$

That leads to relation

$$
\sum_{i \in N} f_{i}(v)=v(N) \sum_{i \in N} p_{N \backslash i}^{i}+\sum_{T \neq \varnothing, T \neq N} v(T)\left[\sum_{i \in T} p_{T \backslash i}^{i}-\frac{1}{|T|+1} \sum_{L:|L|=T T+1, L \supseteq T} \sum_{j \in L} p_{L \backslash j}^{j}\right] .
$$

It comes out that if

$$
\begin{aligned}
& {\left[\sum_{i \in N} p_{N \backslash i}^{i}=1 \text { and } \forall T \notin\{\varnothing, N\}, \forall(i, j) \in N \times N,\right.} \\
& \left.\sum_{i \in T} p_{T \backslash i}^{i}=\frac{1}{|T|+1} \sum_{L:|L|=T \mid+1, L \supseteq T} \sum_{j \in L} p_{L \backslash j}^{j}\right],
\end{aligned}
$$

then $f$ is efficient.

For the converse, following Weber (Weber 1988), for every non empty coali- 
tion $T$, consider both games $v_{T}$ and $\hat{v}_{T}$ defined by:

$$
v_{T}(S)=\left\{\begin{array}{lc}
1 & \text { if } S \supseteq T \\
0 & \text { otherwise }
\end{array}, \hat{v}_{T}(S)=\left\{\begin{array}{lc}
1 & \text { if } S \supsetneqq T \\
0 & \text { otherwise }
\end{array}\right.\right.
$$

Consider the game $v_{N}$, relation 1 becomes

$$
\sum_{i \in N} f_{i}\left(v_{N}\right)=v_{N}(N) \sum_{j \in N} p_{N \backslash j}^{j}+\sum_{S \neq \varnothing, S \neq N} v_{N}(S)\left[\sum_{i \in S} p_{S \backslash i}^{i}-\frac{1}{|S|+1} \sum_{L:|L|=|S|+1, L \supseteq S} \sum_{j \in L} p_{L \backslash j}^{j}\right]
$$

That is $\sum_{i \in N} f_{i}\left(v_{N}\right)=\sum_{j \in N} p_{N \backslash j}^{j}$. Furthermore $\sum_{i \in N} f_{i}\left(v_{N}\right)=1$ since $f$ is efficient. Thus $\sum_{j \in N} p_{N \backslash j}^{j}=1$.

Now for every nonempty coalition $T$ such that $T \neq N$, consider the games $v_{T}$ and $\hat{v}_{T}$. Note that:

$$
\left\{\begin{array}{l}
v_{T}(S)=\hat{v}_{T}(S) \text { for } S \neq T \\
v_{T}(T)=1 \\
\hat{v}_{T}(T)=0
\end{array}\right.
$$

It turns out that $\sum_{i \in N} f_{i}\left(v_{T}\right)-\sum_{i \in N} f_{i}\left(\hat{v}_{T}\right)=\sum_{i \in T} p_{T \backslash i}^{i}-\frac{1}{|T|+1} \sum_{L:|L|=T+1, L \supseteq T} \sum_{j \in L} p_{L \backslash j}^{j}$.

Furthermore $\sum_{i \in N} f_{i}\left(v_{T}\right)=\sum_{i \in N} f_{i}\left(\hat{v}_{T}\right)=1$, since $f$ is efficient.

Therefore $\sum_{i \in T} p_{T \backslash i}^{i}-\frac{1}{|T|+1} \sum_{L:|L|=|T|+1, L \supseteq T} \sum_{j \in L} p_{L \backslash j}^{j}=0$. That ends the proof.

Now we can characterize the solidarity value as a ps-value.

Theorem 13 A ps-value $f$ is the solidarity value, if and only if, $f$ satisfies the symmetry and the efficiency axioms.

Proof. We have $\forall v \in \Gamma, \forall i \in N, f_{i}(v)=\sum_{T / i \in T} p_{T \backslash i}^{i} A^{v}(T)$.

By Theorem 12 we have $\sum_{i \in N} p_{N \backslash i}^{i}=1$ and $\forall T \notin\{\varnothing, N\}$, $\sum_{i \in T} p_{T \backslash i}^{i}=\frac{1}{|T|+1} \sum_{L: L L|=T|+1, L \supseteq T} \sum_{j \in L} p_{L \backslash j}^{j}$. For every $t \in\{0,1, \cdots, n-1\}$, since $f$ is efficient, we have:

$$
\sum_{i \in N} \sum_{T: T \mid=t, i \notin T} p_{T}^{i}=\sum_{i \in N} \sum_{T:|T|=t+1, i \notin T} p_{T}^{i}=\cdots=\sum_{i \in N} \sum_{T:|T|=n-1, i \notin T} p_{T}^{i}=\sum_{i \in N} p_{N \backslash i}^{i}=1
$$

Note that for every $i \in N$, there are $\left(\begin{array}{c}n-1 \\ |T|\end{array}\right)$ coalitions $T$ not containing $i$, and consequently $\left(\begin{array}{c}n-1 \\ |T|\end{array}\right)$ quantities $p_{T}^{i}=p_{|T|}$. Thus, for the $n$ players, there are $n \times\left(\begin{array}{c}n-1 \\ |T|\end{array}\right)$ quantities $p_{T}^{i}$. Note that $\left.\sum_{i \in N} \sum_{T: T \mid=t} p_{T}^{i}=n \times\left(\begin{array}{c}n-1 \\ |T|\end{array}\right) p_{t} \quad{ }^{* \star}\right)$. Both relations $\left({ }^{*}\right)$ and $\left({ }^{* *}\right)$ lead to $n \times\left(\begin{array}{c}n-1 \\ |T|\end{array}\right) p_{t}=1$. Hence $\forall i \in N, \forall T \subseteq N \backslash i$, 


$$
\begin{aligned}
& p_{T}^{i}=p_{t}=\frac{t !(n-t-1) !}{n !} \\
& \text { So, } \forall i \in N, f_{i}(v)=\sum_{T \subseteq N \backslash i} \frac{t !(n-t-1) !}{n !} A^{v}(T \cup i) \text { or } \\
& \forall i \in N, \\
& f_{i}(v)=\sum_{T \mid i \in T} \frac{(t-1) !(n-t) !}{n !} A^{v}(T) .
\end{aligned}
$$

Conversely, using theorem 1, we have the proof.

Recall that the class of probabilistic solidarity values is a generalization of the well known solidarity value of Nowak and Radzik (1994). So they intend to reflect some degree of solidarity among players. The next section focuses on an intuitive solidarity behavior found in literature which we refer as the per-capita productivity behavior. And we identify one subclass of ps-values that exhibits this property.

\section{Probabilistic Solidarity Values and the Per-Capita Productivity Behavior}

First of all, we present the per-capita productivity behavior through the following example found in literature: the set of all players is $N=\{1,2,3\}$ and $v \in \Gamma$ is given by:

$$
v=u_{\{1\}}+2 \cdot u_{\{2,3\}}
$$

where $u_{T}, T \neq \varnothing$ is the unanimity game. There are no synergies between the players in coalitions $\{1\}$ and $\{2,3\}$ and the per-capita productivity in both groups is the same, namely 1 . So, by the per-capita productivity behavior, each solidarity solution for $v$ should reward players so that the per-capita payoff in both groups is also the same. However, various solidarity solutions fail to do so. This is the case for the generalized Solidarity values of Casajus and Huettner (2014) including the Solidarity value of Nowak and Radzik (1994). In fact the Solidarity value $\varphi$ for $v$ is given by $\varphi_{1}(v)=\frac{38}{36}$ and $\varphi_{2}(v)=\varphi_{3}(v)=\frac{35}{36}$ and the per-capita payoff in $\{1\}$ and $\{2,3\}$ is $\frac{38}{36}$ and $\frac{35}{36}$ respectively.

In contrast ps-values provide an infinite set of solutions satisfying the per-capita productivity behavior. To see this, look at the following example: $N=\{1,2,3\}$ and $v \in \Gamma$ is given by:

$$
v(\{1,2\})=v(\{1\})+\{2\}, v(\{1,3\})=v(\{1\})+\{3\}, v(\{2,3\})=2 v(\{1\}) .
$$

One can easily checks that both groups $\{1\}$ and $\{2,3\}$ have independent productivities and the same per-capita productivity. Now consider the set of all ps-values $\phi$ satisfying:

$$
p_{\varnothing}^{1}=p_{\varnothing}^{2}=p_{\varnothing}^{3}=\frac{1}{3}
$$




$$
\begin{gathered}
p_{\{1\}}^{2}=\frac{1}{7}, p_{\{2\}}^{1}=\frac{4}{21}, p_{\{1\}}^{3}=\frac{1}{7}, p_{\{3\}}^{1}=\frac{4}{21}, p_{\{3\}}^{2}+p_{\{2\}}^{3}=\frac{1}{3} \\
p_{\{2,3\}}^{1}=\frac{2}{7}, p_{\{1,3\}}^{2}+p_{\{1,2\}}^{3}=\frac{5}{7}
\end{gathered}
$$

We have:

$$
\begin{aligned}
& \phi_{1}(v)= {\left[p_{\varnothing}^{1}+\frac{1}{2} p_{\{2\}}^{1}+\frac{1}{2} p_{\{3\}}^{1}+\frac{5}{3} p_{\{2,3\}}^{1}\right] v(1)+\left[\frac{1}{2} p_{\{2\}}^{1}-\frac{1}{3} p_{\{2,3\}}^{1}\right] v(2) } \\
&+\left[\frac{1}{2} p_{\{3\}}^{1}-\frac{1}{3} p_{\{2,3\}}^{1}\right] v(3) \\
& \phi_{2}(v)=\left[\frac{1}{2} p_{\{1\}}^{2}+2 p_{\{3\}}^{2}+\frac{5}{3} p_{\{1,3\}}^{2}\right] v(1)+\left[p_{\varnothing}^{2}+\frac{1}{2} p_{\{1\}}^{2}-\frac{1}{2} p_{\{3\}}^{2}-\frac{1}{3} p_{\{1,3\}}^{2}\right] v(2) \\
&+\left[-\frac{1}{2} p_{\{3\}}^{2}-\frac{1}{3} p_{\{1,3\}}^{2}\right] v(3) \\
& \phi_{3}(v)=\left[\frac{1}{2} p_{\{1\}}^{3}+2 p_{\{2\}}^{3}+\frac{5}{3} p_{\{1,2\}}^{3}\right] v(1)+\left[-\frac{1}{2} p_{\{2\}}^{3}-\frac{1}{3} p_{\{1,2\}}^{3}\right] v(2) \\
&+\left[p_{\varnothing}^{3}+\frac{1}{2} p_{\{1\}}^{3}-\frac{1}{2} p_{\{2\}}^{3}-\frac{1}{3} p_{\{1,2\}}^{3}\right] v(3)
\end{aligned}
$$

It is clear that $2 \phi_{1}(v)-\left[\phi_{2}(v)+\phi_{3}(v)\right]=0$. So each $\phi$ satisfies the per-capita productivity behavior. And by theorem 12, every $\phi$ described above is efficient.

Now before characterizing symmetric ps-values which exhibit the per-capita productivity behavior, we need to state the property.

Axiom $4 A$ value $\varphi$ satisfies the per-capita productivity behavior when for all games $v$ and $T \subseteq N, T \neq \varnothing, N$ :

$$
\begin{aligned}
& \text { If } \forall S \subseteq N, v(S)=v(S \cap T)+v(S \cap(N \backslash T)) \text { and } \frac{v(T)}{|T|}=\frac{v(N \backslash T)}{|N \backslash T|} \text {, then } \\
& \frac{\sum_{i \in T} \varphi_{i}(v)}{|T|}=\frac{\sum_{i \in N \backslash T} \varphi_{i}(v)}{|N \backslash T|} .
\end{aligned}
$$

Based on the example above, we consider $v \in \Gamma$, a symmetric ps-value $\phi$ and for each player $i$, we express $\phi_{i}(v)$ according to $v\left(T_{0}\right), T_{0} \subseteq T \cup(N \backslash T)$, $T \neq N \backslash T$.

From now on, let us consider $V$ and $T$ as described in axiom 4, and $\phi$ a symmetric ps-value. Moreover assume that $|T| \leq|N \backslash T|$. Also for every $i \in N$ and $T_{0} \subseteq T \cup(N \backslash T),\left(\phi_{i}(v)\right)_{T_{0}}$ is the estimation of $\phi_{i}(v)$ on $v\left(T_{0}\right)$. And due to theorem $4, p_{t}$ denotes the probability to join a coalition of size $t$ where $t=0,1,2, \cdots,|N|-1$. Notice that considering the definition of a ps-value and the conclusion in axiom 4 , it is sufficient for the payoff $\phi_{i}(v)$, to simply write terms using probabilities from $p_{0}$ to $p_{|N|-2}$.

Lemma 14 Let us consider $T_{0} \varsubsetneqq T$ and $i \in T_{0}$ : 


$$
\left(\phi_{i}(v)\right)_{T_{0}}=\left[p_{\left|T_{0}\right|-1}-\frac{|T|-\left|T_{0}\right|}{\left|T_{0}\right|+1} p_{\left|T_{0}\right|}+\sum_{k=1}^{|N \backslash T|} \frac{\left|T_{0}\right|\left(\begin{array}{c}
|N|-|T| \\
k
\end{array}\right)}{\left|T_{0}\right|+k} p_{\left|T_{0}\right|+k-1}\right.
$$

a)

$$
\left.-\sum_{k=1}^{|N|-|T|} \frac{\left(|T|-\left|T_{0}\right|\right)\left(\begin{array}{c}
|N|-|T| \\
k
\end{array}\right)}{\left|T_{0}\right|+k+1} p_{\left|T_{0}\right|+k}\right] v\left(T_{0}\right)
$$

$\left|T \backslash T_{0}\right|>1$.

b)

$$
\begin{aligned}
& \left(\phi_{i}(v)\right)_{T_{0}}=\left[p_{\left|T_{0}\right|-1}-\frac{1}{\left|T_{0}\right|+1} p_{\left|T_{0}\right|}+\sum_{k=1}^{|N \backslash T|} \frac{\left|T_{0}\right|\left(\begin{array}{c}
|N|-|T| \\
k
\end{array}\right)}{\left|T_{0}\right|+k} p_{\left|T_{0}\right|+k-1}\right. \\
& \left.-\sum_{k=1}^{|N|-|T|-1} \frac{\left(\begin{array}{c}
|N|-|T| \\
k
\end{array}\right)}{\left|T_{0}\right|+k+1} p_{\left|T_{0}\right|+k}\right] v\left(T_{0}\right)
\end{aligned}
$$

We must point out that in all the following proofs, we might write a quantity other than $v\left(T_{0}\right)$, in order to make reading more comfortable.

Proof. Recall that $\phi_{i}(v)=\sum_{T / i \notin T} p_{T}^{i} A^{v}(T \cup i)$. So, excluding other quantities present in $A^{v}(T \cup i)$, we obtain:

$$
\begin{aligned}
& \left(\phi_{i}(v)\right)_{T_{0}}=\frac{1}{\left|T_{0}\right|} p_{T_{0} \backslash\{i\}}^{i}\left[\left|T_{0}\right| v\left(T_{0}\right)\right] \\
& +\sum_{i_{1} \in T \backslash T_{0}} \frac{1}{\left|T_{0}\right|+1} p_{T_{0} \backslash\left\{\{\} \cup\left\{i_{1}\right\}\right.}^{i}\left[\left(\left|T_{0}\right|+1\right) v\left(T_{0} \cup\left\{i_{1}\right\}\right)-v\left(T_{0}\right)\right] \\
& \text { a) }+\sum_{i_{1} \in T \backslash T_{0}} \sum_{k=1}^{|N|-|T|} \sum_{\tilde{T} \subset N \backslash T} \frac{1}{|\tilde{T}|=k} \mid p_{0}^{i} p_{T_{0} \backslash\left\{\{\} \cup \cup \tilde{T} \cup\left\{i_{1}\right\}\right.}\left[\left(\left|T_{0}\right|+k+1\right) v\left(T_{0} \cup \tilde{T} \bigcup\left\{i_{1}\right\}\right)\right.
\end{aligned}
$$

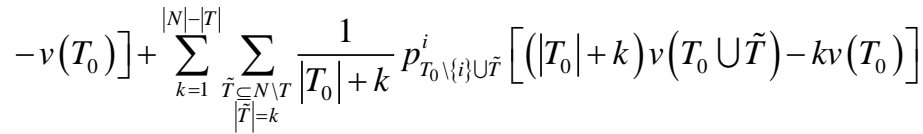

or:

$$
\begin{aligned}
\left(\phi_{i}(v)\right)_{T_{0}}= & p_{\left|T_{0}\right|-1} v\left(T_{0}\right)-\frac{\left(|T|-\left|T_{0}\right|\right)}{\left|T_{0}\right|+1} p_{\left|T_{0}\right|} v\left(T_{0}\right) \\
& -\sum_{k=1}^{|N|-|T|} \frac{\left(|T|-\left|T_{0}\right|\right)\left(\begin{array}{c}
|N|-|T| \\
k
\end{array}\right)}{\left|T_{0}\right|+k+1} p_{\left|T_{0}\right|+k} v\left(T_{0}\right) \\
& +\sum_{k=1}^{|N|-|T|} \frac{\left|T_{0}\right|\left(\begin{array}{c}
|N|-|T| \\
k
\end{array}\right)}{\left|T_{0}\right|+k} p_{\left|T_{0}\right|+k-1} v\left(T_{0}\right) .
\end{aligned}
$$




$$
\begin{aligned}
& \left(\phi_{i}(v)\right)_{T_{0}}=\frac{1}{\left|T_{0}\right|} p_{T_{0} \backslash\{i\}}^{i}\left[\left|T_{0}\right| v\left(T_{0}\right)\right] \\
& +\sum_{i_{1} \in T \backslash T_{0}} \frac{1}{\left|T_{0}\right|+1} p_{T_{0} \backslash\left\{\{i\} \cup\left\{i_{1}\right\}\right.}^{i}\left[\left(\left|T_{0}\right|+1\right) v\left(T_{0} \cup\left\{i_{1}\right\}\right)-v\left(T_{0}\right)\right] \\
& \text { b) }+\sum_{i_{1} \in T \backslash T_{0}} \sum_{k=1}^{|N|-|T|-1} \sum_{\substack{C \\
\widetilde{T} \mid=k}} \frac{1}{\left|T_{0}\right|+k+1} p_{T_{0} \backslash\left\{\{\} \cup \cup \tilde{T} \cup\left\{i_{1}\right\}\right.}^{i}\left[\left(\left|T_{0}\right|+k+1\right) v\left(T_{0} \cup \tilde{T} \bigcup\left\{i_{1}\right\}\right)\right. \\
& \left.-v\left(T_{0}\right)\right]+\sum_{k=1}^{|N|-|T|} \sum_{\substack{\tilde{T} \subset N \backslash T \\
|\tilde{T}|=k}} \frac{1}{\left|T_{0}\right|+k} p_{T_{0} \backslash\{i\} \cup \tilde{T}}^{i}\left[\left(\left|T_{0}\right|+k\right) v\left(T_{0} \cup \tilde{T}\right)-k v\left(T_{0}\right)\right] \\
& \left(\phi_{i}(v)\right)_{T_{0}}=p_{\left|T_{0}\right|-1} v\left(T_{0}\right)-\frac{1}{\left|T_{0}\right|+1} p_{\left|T_{0}\right|} v\left(T_{0}\right) \\
& \text { or } \\
& -\sum_{k=1}^{|N|-|T|-1} \frac{\left(\begin{array}{c}
|N|-|T| \\
k
\end{array}\right)}{\left|T_{0}\right|+k+1} p_{\left|T_{0}\right|+k} v\left(T_{0}\right) \\
& +\sum_{k=1}^{|N|-|T|} \frac{\left.\left|T_{0}\right| \begin{array}{c}
|N|-|T| \\
k
\end{array}\right)}{\left|T_{0}\right|+k} p_{\left|T_{0}\right|+k-1} v\left(T_{0}\right) .
\end{aligned}
$$

Lemma 15 Consider $T_{0} \varsubsetneqq T$ and $i \in T \backslash T_{0}$ :

a) $\left(\phi_{i}(v)\right)_{T_{0}}=\left[-\frac{1}{\left|T_{0}\right|+1} p_{\left|T_{0}\right|}-\sum_{k=1}^{|N|-|T|} \frac{\left(\begin{array}{c}|N|-|T| \\ k\end{array}\right)}{\left|T_{0}\right|+k+1} p_{\left|T_{0}\right|+k}\right] v\left(T_{0}\right)$, when $\left|T \backslash T_{0}\right|>1$.

b) $\left(\phi_{i}(v)\right)_{T_{0}}=\left[-\frac{1}{\left|T_{0}\right|+1} p_{\left|T_{0}\right|}-\sum_{k=1}^{|N|-|T|-1} \frac{\left(\begin{array}{c}|N|-|T| \\ k\end{array}\right)}{\left|T_{0}\right|+k+1} p_{\left|T_{0}\right|+k}\right] v\left(T_{0}\right), \quad\left|T \backslash T_{0}\right|=1$.

$$
\left(\phi_{i}(v)\right)_{T_{0}}=\frac{1}{\left|T_{0}\right|+1} p_{T_{0}}^{i}\left[\left(\left|T_{0}\right|+1\right) v\left(T_{0} \cup\{i\}\right)-v\left(T_{0}\right)\right]
$$

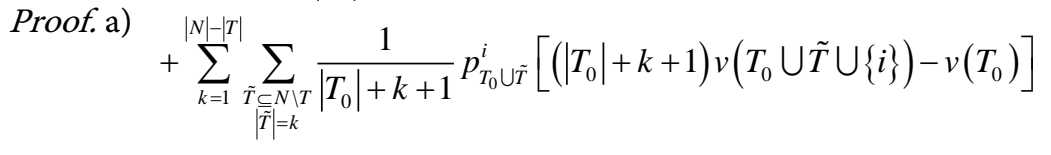

thus $\left(\phi_{i}(v)\right)_{T_{0}}=-\frac{1}{\left|T_{0}\right|+1} p_{\left|T_{0}\right|} v\left(T_{0}\right)-\sum_{k=1}^{|N|-|T|} \frac{\left(\begin{array}{c}|N|-|T| \\ k\end{array}\right)}{\left|T_{0}\right|+k+1} p_{\left|T_{0}\right|+k} v\left(T_{0}\right)$.

$$
\left(\phi_{i}(v)\right)_{T_{0}}=\frac{1}{\left|T_{0}\right|+1} p_{T_{0}}^{i}\left[\left(\left|T_{0}\right|+1\right) v\left(T_{0} \cup\{i\}\right)-v\left(T_{0}\right)\right]
$$

b) $+\sum_{k=1}^{|N|-|T|-1} \sum_{\substack{\tilde{T} \subset N \backslash T \\|\tilde{T}|=k}} \frac{1}{\left|T_{0}\right|+k+1} p_{T_{0} \cup \tilde{T}}^{i}\left[\left(\left|T_{0}\right|+k+1\right) v\left(T_{0} \bigcup \tilde{T} \bigcup\{i\}\right)-v\left(T_{0}\right)\right]$

or $\left(\phi_{i}(v)\right)_{T_{0}}=-\frac{1}{\left|T_{0}\right|+1} p_{\left|T_{0}\right|} v\left(T_{0}\right)-\sum_{k=1}^{|N|-|T|-1} \frac{\left(\begin{array}{c}|N|-|T| \\ k\end{array}\right)}{\left|T_{0}\right|+k+1} p_{\left|T_{0}\right|+k} v\left(T_{0}\right)$. 
Lemma 16 For $T_{0}=T$ and $i \in T_{0}$ :

$$
\begin{aligned}
& \left(\phi_{i}(v)\right)_{T_{0}}=\left[p_{\left|T_{0}\right|-1}+\sum_{k=1}^{|N|-|T|-1} \frac{\left|T_{0}\right|\left(\begin{array}{c}
|N|-|T| \\
k
\end{array}\right)}{\left|T_{0}\right|+k} p_{\left|T_{0}\right|+k-1}\right. \\
& \left.+\sum_{k=0}^{\left|T_{0}\right|-2} \frac{|N \backslash T|^{2}\left(\begin{array}{c}
\left|T_{0}\right|-1 \\
k
\end{array}\right)}{|T|[|N \backslash T|+k+1]} p_{|N \backslash T|+k}\right] v\left(T_{0}\right) \\
& \left(\phi_{i}(v)\right)_{T_{0}}=\frac{1}{\left|T_{0}\right|} p_{T_{0} \backslash\{i\}}^{i}\left[\left|T_{0}\right| v\left(T_{0}\right)\right] \\
& +\sum_{k=1}^{|N|-|T|-1} \sum_{\substack{\tilde{T} \subset N \backslash T \\
|\widetilde{T}|=k}} \frac{1}{\left|T_{0}\right|+k} p_{T_{0} \backslash\{i\} \cup \cup \tilde{T}}^{i}\left[\left(\left|T_{0}\right|+k\right) v\left(T_{0} \cup \tilde{T}\right)-k v\left(T_{0}\right)\right] \\
& +\sum_{k=0}^{\left|T_{0}\right|-2} \sum_{\substack{\tilde{T}_{\subset T^{\prime} \backslash\{\langle i\}} \\
|\tilde{T}|=k}} \frac{1}{|N \backslash T|+k+1} p_{N \backslash T \cup \tilde{T}}^{i}[(|N \backslash T|+k+1) v(N \backslash T \bigcup \tilde{T} \bigcup\{i\}) \\
& -(k+1) v(N \backslash T)]=p_{\left|T_{0}\right|-1} v\left(T_{0}\right)+\sum_{k=1}^{|N|-|T|-1} \frac{\left|T_{0}\right|\left(\begin{array}{c}
|N|-|T| \\
k
\end{array}\right)}{\left|T_{0}\right|+k} p_{\left|T_{0}\right|+k-1} v\left(T_{0}\right) \\
& +\sum_{k=0}^{\left|T_{0}\right|-2} \sum_{\tilde{T} \subset T_{0} \backslash\{i\}} \frac{|N \backslash T|\left(\begin{array}{c}
\left|T_{0}\right|-1 \\
k
\end{array}\right)}{|N \backslash T|+k+1} p_{|N \backslash T|+k} v(N \backslash T)
\end{aligned}
$$

Proof.

Hence

$$
\left(\phi_{i}(v)\right)_{T_{0}}=p_{\left|T_{0}\right|-1} v\left(T_{0}\right)+\sum_{k=1}^{|N|-|T|-1 \mid} \frac{\left|T_{0}\right|\left(\begin{array}{c}
|N|-|T| \\
k
\end{array}\right)}{\left|T_{0}\right|+k} p_{\left|T_{0}\right|+k-1} v\left(T_{0}\right)
$$

$$
+\sum_{k=0}^{\left|T_{0}\right|-2} \frac{|N \backslash T|^{2}\left(\begin{array}{c}
\left|T_{0}\right|-1 \\
k
\end{array}\right)}{|T|[|N \backslash T|+k+1]} p_{|N \backslash T|+k} v\left(T_{0}\right)
$$

Lemma 17 Consider $T_{0} \varsubsetneqq N \backslash T$ and $i \in T$ :

a)

$$
\left(\phi_{i}(v)\right)_{T_{0}}=\left[\frac{\left|T_{0}\right|}{\left|T_{0}\right|+1} p_{\left|T_{0}\right|}+\sum_{k=1}^{|T|-1} \frac{\left|T_{0}\right|\left(\begin{array}{c}
|T|-1 \\
k
\end{array}\right)}{\left|T_{0}\right|+k+1} p_{\left|T_{0}\right|+k}\right.
$$

$$
\left.-\sum_{k=1}^{|T|-1} \frac{\left(|N|-|T|-\left|T_{0}\right|\right)\left(\begin{array}{c}
|T|-1 \\
k
\end{array}\right)}{\left|T_{0}\right|+k+2} p_{\left|T_{0}\right|+k+1}-\frac{\left(|N|-|T|-\left|T_{0}\right|\right)}{\left|T_{0}\right|+2} p_{\left|T_{0}\right|+1}\right] v\left(T_{0}\right)
$$

when $\left(|N|-|T|-\left|T_{0}\right|\right)>1$. 
b)

$$
\begin{aligned}
& \left(\phi_{i}(v)\right)_{T_{0}}=\left[\frac{\left|T_{0}\right|}{\left|T_{0}\right|+1} p_{\left|T_{0}\right|}+\sum_{k=1}^{|T|-1} \frac{\left|T_{0}\right|\left(\begin{array}{c}
|T|-1 \\
k
\end{array}\right)}{\left|T_{0}\right|+k+1} p_{\left|T_{0}\right|+k}-\sum_{k=0}^{|T|-2} \frac{\left(\begin{array}{c}
|T|-1 \\
k
\end{array}\right)}{\left|T_{0}\right|+k+2} p_{\left|T_{0}\right|+k+1}\right] v\left(T_{0}\right), \\
& \text { when }\left(|N|-|T|-\left|T_{0}\right|\right)=1 .
\end{aligned}
$$

Proof. a)

$$
\begin{aligned}
& \left(\phi_{i}(v)\right)_{T_{0}}=\frac{1}{\left|T_{0}\right|+1} p_{T_{0}}^{i}\left[\left(\left|T_{0}\right|+1\right) v\left(T_{0} \cup\{i\}\right)-v\left(T_{0}\right)\right]
\end{aligned}
$$

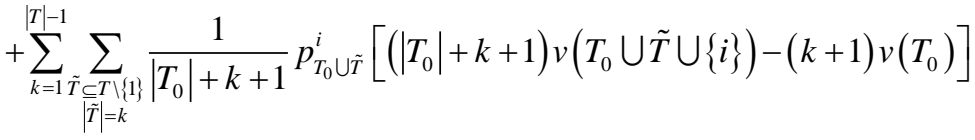

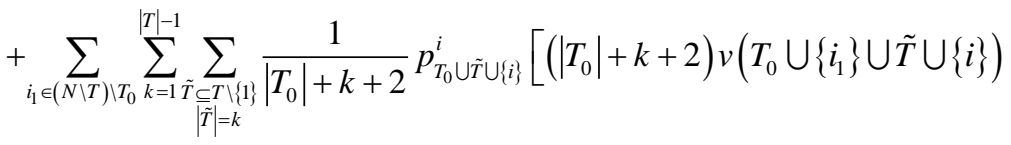

$$
\begin{aligned}
& \left.-v\left(T_{0}\right)\right]+\sum_{i_{1} \in(N \backslash T) \backslash T_{0}} \frac{1}{\left|T_{0}\right|+2} p_{T_{0} \cup\{i\}}^{i}\left[\left(\left|T_{0}\right|+2\right) v\left(T_{0} \bigcup\left\{i_{1}\right\} \bigcup\{i\}\right)-v\left(T_{0}\right)\right] \\
& \left(\phi_{i}(v)\right)_{T_{0}}=\frac{\left|T_{0}\right|}{\left|T_{0}\right|+1} p_{\left|T_{0}\right|} v\left(T_{0}\right)+\sum_{k=1}^{|T|-1} \frac{\left|T_{0}\right|\left(\begin{array}{c}
|T|-1 \\
k
\end{array}\right)}{\left|T_{0}\right|+k+1} p_{\left|T_{0}\right|+k} v\left(T_{0}\right) \\
& -\sum_{k=1}^{|T|-1} \frac{\left(|N|-|T|-\left|T_{0}\right|\right)\left(\begin{array}{c}
|T|-1 \\
k
\end{array}\right)}{\left|T_{0}\right|+k+2} p_{\left|T_{0}\right|+k+1} v\left(T_{0}\right) \\
& -\frac{\left(|N|-|T|-\left|T_{0}\right|\right)}{\left|T_{0}\right|+2} p_{\left|T_{0}\right|+1} v\left(T_{0}\right) \\
& \left(\phi_{i}(v)\right)_{T_{0}}=\frac{1}{\left|T_{0}\right|+1} p_{T_{0}}^{i}\left[\left(\left|T_{0}\right|+1\right) v\left(T_{0} \cup\{i\}\right)-v\left(T_{0}\right)\right] \\
& +\sum_{k=1}^{|T|-1} \sum_{\tilde{T} \subset T \backslash\{\{1\}} \frac{1}{\left|T_{0}\right|+k+1} p_{T_{0} \cup \tilde{T}}^{i}\left[\left(\left|T_{0}\right|+k+1\right) v\left(T_{0} \bigcup \tilde{T} \bigcup\{i\}\right)-(k+1) v\left(T_{0}\right)\right]
\end{aligned}
$$

$$
\begin{aligned}
& +\sum_{i_{1} \in(N \backslash T) \backslash T_{0}} \sum_{k=0}^{|T|-2} \sum_{\tilde{T} \in T \backslash\{1\}} \frac{1}{\left|T_{0}\right|+k+2} p_{T_{0} \cup \tilde{T} \cup\left\{i_{1}\right\}}^{i}\left[( | T _ { 0 } | + k + 2 ) v \left(T_{0} \bigcup\left\{i_{1}\right\} \bigcup \tilde{T}\right.\right. \\
& \left.\cup\{i\})-v\left(T_{0}\right)\right]
\end{aligned}
$$

Hence

$$
\left(\phi_{i}(v)\right)_{T_{0}}=\frac{\left|T_{0}\right|}{\left|T_{0}\right|+1} p_{\left|T_{0}\right|} v\left(T_{0}\right)+\sum_{k=1}^{|T|-1} \frac{\left|T_{0}\right|\left(\begin{array}{c}
|T|-1 \\
k
\end{array}\right)}{\left|T_{0}\right|+k+1} p_{\left|T_{0}\right|+k} v\left(T_{0}\right)
$$

$$
-\sum_{k=0}^{|T|-2} \frac{\left(\begin{array}{c}
|T|-1 \\
k
\end{array}\right)}{\left|T_{0}\right|+k+2} p_{\left|T_{0}\right|+k+1} v\left(T_{0}\right)
$$

Lemma 18 For $T_{0} \varsubsetneqq N \backslash T$ and $i \in(N \backslash T) \backslash T_{0}$ : 
a) $\left(\phi_{i}(v)\right)_{T_{0}}=\left[\frac{-1}{\left|T_{0}\right|+1} p_{\left|T_{0}\right|}-\sum_{k=1}^{|T|} \frac{\left(\begin{array}{c}|T| \\ k\end{array}\right)}{\left|T_{0}\right|+k+1} p_{\left|T_{0}\right|+k}\right] v\left(T_{0}\right), \quad\left(|N|-|T|-\left|T_{0}\right|\right)>1$.

b) $\left(\phi_{i}(v)\right)_{T_{0}}=\left[\frac{-1}{\left|T_{0}\right|+1} p_{\left|T_{0}\right|}-\sum_{k=1}^{|T|-1} \frac{\left(\begin{array}{c}|T| \\ k\end{array}\right)}{\left|T_{0}\right|+k+1} p_{\left|T_{0}\right|+k}\right] v\left(T_{0}\right), \quad\left(|N|-|T|-\left|T_{0}\right|\right)=1$.

$$
\left(\phi_{i}(v)\right)_{T_{0}}=\frac{1}{\left|T_{0}\right|+1} p_{T_{0}}^{i}\left[\left(\left|T_{0}\right|+1\right) v\left(T_{0} \cup\{i\}\right)-v\left(T_{0}\right)\right]
$$

Proof. a) $+\sum_{\substack{k=1 \\|T \backslash T\\| \tilde{T} \mid=k}}^{|T|} \frac{1}{\left|T_{0}\right|+k+1} p_{T_{0} \cup \tilde{T}}^{i}\left[\left(\left|T_{0}\right|+k+1\right) v\left(T_{0} \cup \tilde{T} \bigcup\{i\}\right)-v\left(T_{0}\right)\right]$

So $\left(\phi_{i}(v)\right)_{T_{0}}=-\frac{1}{\left|T_{0}\right|+1} p_{\left|T_{0}\right|} v\left(T_{0}\right)-\sum_{k=1}^{|T|} \frac{\left(\begin{array}{c}|T| \\ k\end{array}\right)}{\left|T_{0}\right|+k+1} p_{\left|T_{0}\right|+k} v\left(T_{0}\right)$.

$$
\left(\phi_{i}(v)\right)_{T_{0}}=\frac{1}{\left|T_{0}\right|+1} p_{T_{0}}^{i}\left[\left(\left|T_{0}\right|+1\right) v\left(T_{0} \cup\{i\}\right)-v\left(T_{0}\right)\right]
$$

b) $+\sum_{k=1}^{|T|-1} \sum_{\substack{T \\|\tilde{T}|=k}} \frac{1}{\left|T_{0}\right|+k+1} p_{T_{0} \cup \tilde{T}}^{i}\left[\left(\left|T_{0}\right|+k+1\right) v\left(T_{0} \cup \tilde{T} \bigcup\{i\}\right)-v\left(T_{0}\right)\right]$

Thus $\left(\phi_{i}(v)\right)_{T_{0}}=-\frac{1}{\left|T_{0}\right|+1} p_{\left|T_{0}\right|} v\left(T_{0}\right)-\sum_{k=1}^{|T|-1} \frac{\left(\begin{array}{c}|T| \\ k\end{array}\right)}{\left|T_{0}\right|+k+1} p_{\left|T_{0}\right|+k} v\left(T_{0}\right)$.

Lemma 19 Take $T_{0} \varsubsetneqq N \backslash T$ and $i \in T_{0}$ :

a)

$$
\left(\phi_{i}(v)\right)_{T_{0}}=\left[p_{\left|T_{0}\right|-1}-\frac{\left(|N|-|T|-\left|T_{0}\right|\right)}{\left|T_{0}\right|+1} p_{\left|T_{0}\right|}+\sum_{k=1}^{|T|} \frac{\left|T_{0}\right|\left(\begin{array}{c}
|T| \\
k
\end{array}\right)}{\left|T_{0}\right|+k} p_{\left|T_{0}\right|+k-1}\right.
$$

$$
\left.-\sum_{k=1}^{|T|} \frac{\left(|N|-|T|-\left|T_{0}\right|\right)\left(\begin{array}{c}
|T| \\
k
\end{array}\right)}{\left|T_{0}\right|+k+1} p_{\left|T_{0}\right|+k}\right] v\left(T_{0}\right)
$$

when $\left(|N|-|T|-\left|T_{0}\right|\right)>1$.

b)

$\left(\phi_{i}(v)\right)_{T_{0}}=\left[p_{\left|T_{0}\right|-1}-\frac{1}{\left|T_{0}\right|+1} p_{\left|T_{0}\right|}+\sum_{k=1}^{|T|} \frac{\left|T_{0}\right|\left(\begin{array}{c}|T| \\ k\end{array}\right)}{\left|T_{0}\right|+k} p_{\left|T_{0}\right|+k-1}-\sum_{k=1}^{|T|-1} \frac{\left(\begin{array}{c}|T| \\ k\end{array}\right)}{\left|T_{0}\right|+k+1} p_{\left|T_{0}\right|+k}\right] v\left(T_{0}\right)$,

with $\left(|N|-|T|-\left|T_{0}\right|\right)=1$. 


$$
\begin{aligned}
& \left(\phi_{i}(v)\right)_{T_{0}}=\frac{1}{\left|T_{0}\right|} p_{T_{0} \backslash\{i\}}^{i}\left[\left|T_{0}\right| v\left(T_{0}\right)\right] \\
& +\sum_{i_{1} \in(N \backslash T) \backslash T_{0}} \frac{1}{\left|T_{0}\right|+1} p_{T_{0} \backslash\{i\} \cup\left\{i_{1}\right\}}^{i}\left[\left(\left|T_{0}\right|+1\right) v\left(T_{0} \bigcup\left\{i_{1}\right\}\right)-v\left(T_{0}\right)\right] \\
& \text { Proof. a) }+\sum_{k=1}^{|T|} \sum_{\substack{T \\
|\tilde{T}|=k}} \frac{1}{\left|T_{0}\right|+k} p_{T_{0} \backslash\{i\} \cup \tilde{T}}^{i}\left[\left(\left|T_{0}\right|+k\right) v\left(T_{0} \cup \tilde{T}\right)-k v\left(T_{0}\right)\right]
\end{aligned}
$$

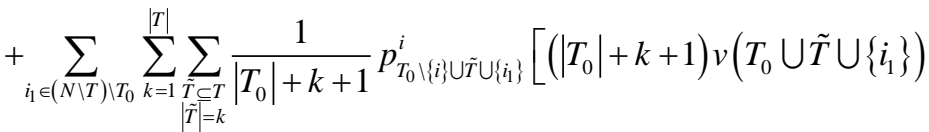

$$
\begin{aligned}
& \left.-v\left(T_{0}\right)\right] \\
& \left(\phi_{i}(v)\right)_{T_{0}}=p_{\left|T_{0}\right|-1} v\left(T_{0}\right)-\frac{\left(|N|-|T|-\left|T_{0}\right|\right)}{\left|T_{0}\right|+1} p_{\left|T_{0}\right|} v\left(T_{0}\right)
\end{aligned}
$$

That is

$$
+\sum_{k=1}^{|T|} \frac{\left|T_{0}\right|\left(\begin{array}{c}
(T \mid \\
k
\end{array}\right)}{\left|T_{0}\right|+k} p_{\left|T_{0}\right|+k-1} v\left(T_{0}\right)-\sum_{k=1}^{|T|} \frac{\left(|N|-|T|-\left|T_{0}\right|\right)\left(\begin{array}{c}
|T| \\
k
\end{array}\right)}{\left|T_{0}\right|+k+1} p_{\left|T_{0}\right|+k} v\left(T_{0}\right)
$$$$
\left(\phi_{i}(v)\right)_{T_{0}}=\frac{1}{\left|T_{0}\right|} p_{T_{0} \backslash\{i\}}^{i}\left[\left|T_{0}\right| v\left(T_{0}\right)\right]
$$$$
+\sum_{i_{1} \in(N \backslash T) \backslash T_{0}} \frac{1}{\left|T_{0}\right|+1} p_{T_{0} \backslash\left\{\{\} \cup\left\{i_{1}\right\}\right.}^{i}\left[\left(\left|T_{0}\right|+1\right) v\left(T_{0} \cup\left\{i_{1}\right\}\right)-v\left(T_{0}\right)\right]
$$

b) $+\sum_{k=1}^{|T|} \sum_{\substack{T \in T \\ \mid \tilde{T}=k}} \frac{1}{T_{0} \mid+k} p_{T_{0} \backslash\{i\} \cup \tilde{T}}^{i}\left[\left(\left|T_{0}\right|+k\right) v\left(T_{0} \cup \tilde{T}\right)-k v\left(T_{0}\right)\right]$

$$
\begin{aligned}
& +\sum_{i_{1} \in(N \backslash T) \backslash T_{0}} \sum_{k=1}^{|T|-1} \sum_{\substack{\tilde{T} \in T \\
|\tilde{T}|=k}} \frac{1}{\left|T_{0}\right|+k+1} p_{T_{0} \backslash\{i\} \cup \tilde{T} \cup\left\{i_{1}\right\}}^{i}\left[\left(\left|T_{0}\right|+k+1\right) v\left(T_{0} \cup \tilde{T} \cup\left\{i_{1}\right\}\right)\right. \\
& \left.-v\left(T_{0}\right)\right]
\end{aligned}
$$

And

$$
\left(\phi_{i}(v)\right)_{T_{0}}=p_{\left|T_{0}\right|-1} v\left(T_{0}\right)-\frac{1}{\left|T_{0}\right|+1} p_{\left|T_{0}\right|} v\left(T_{0}\right)+\sum_{k=1}^{|T|} \frac{\left.\left|T_{0}\right| \begin{array}{c}
|T| \\
k
\end{array}\right)}{\left|T_{0}\right|+k} p_{\left|T_{0}\right|+k-1} v\left(T_{0}\right)
$$

$$
-\sum_{k=1}^{|T|-1} \frac{\left(\begin{array}{c}
|T| \\
k
\end{array}\right)}{\left|T_{0}\right|+k+1} p_{\left|T_{0}\right|+k} v\left(T_{0}\right)
$$

Lemma 20 Consider $T_{0} \varsubsetneqq T$ and $i \in N \backslash T$ :

a)

$$
\left(\phi_{i}(v)\right)_{T_{0}}=\left[\frac{\left|T_{0}\right|}{\left|T_{0}\right|+1} p_{\left|T_{0}\right|}-\frac{\left(|T|-\left|T_{0}\right|\right)}{\left|T_{0}\right|+2} p_{\left|T_{0}\right|+1}+\sum_{k=1}^{|N \backslash T|-1} \frac{\left|T_{0}\right|\left(\begin{array}{c}
|N \backslash T|-1 \\
k
\end{array}\right)}{\left|T_{0}\right|+k+1} p_{\left|T_{0}\right|+k}\right.
$$

$$
\left.-\sum_{k=1}^{|N \backslash T|-1} \frac{\left(|T|-\left|T_{0}\right|\right)\left(\begin{array}{c}
|N \backslash T|-1 \\
k
\end{array}\right)}{\left|T_{0}\right|+k+2} p_{\left|T_{0}\right|+k+1}\right] v\left(T_{0}\right)
$$


when $|T|-\left|T_{0}\right|>1$.

b)

$$
\left(\phi_{i}(v)\right)_{T_{0}}=\left[\frac{\left|T_{0}\right|}{\left|T_{0}\right|+1} p_{\left|T_{0}\right|}-\frac{1}{\left|T_{0}\right|+2} p_{\left|T_{0}\right|+1}+\sum_{k=1}^{|N \backslash T|-1} \frac{\left|T_{0}\right|\left(\begin{array}{c}
|N \backslash T|-1 \\
k
\end{array}\right)}{\left|T_{0}\right|+k+1} p_{\left|T_{0}\right|+k}\right.
$$

$$
\left.-\sum_{k=1}^{|N \backslash T|-2} \frac{\left(\begin{array}{c}
|N \backslash T|-1 \\
k
\end{array}\right)}{\left|T_{0}\right|+k+2} p_{\left|T_{0}\right|+k+1}\right] v\left(T_{0}\right)
$$

, with

$|T|-\left|T_{0}\right|=1$.

$$
\begin{aligned}
& \left(\phi_{i}(v)\right)_{T_{0}}=\frac{1}{\left|T_{0}\right|+1} p_{T_{0}}^{i}\left[\left(\left|T_{0}\right|+1\right) v\left(T_{0} \cup\{i\}\right)-v\left(T_{0}\right)\right] \\
& +\sum_{i_{1} \in T \backslash T_{0}} \frac{1}{\left|T_{0}\right|+2} p_{T_{0} \cup\left\{\left\{_{1}\right\}\right.}^{i}\left[\left(\left|T_{0}\right|+2\right) v\left(T_{0} \bigcup\left\{i_{1}\right\} \bigcup\{i\}\right)-v\left(T_{0}\right)\right]
\end{aligned}
$$

Proof. a) $+\sum_{k=1}^{|N| T \mid-1} \sum_{\substack{\tilde{T} \subseteq(N \backslash T) \backslash\{i\} \\|\tilde{T}|=k}} \frac{1}{\left|T_{0}\right|+k+1} p_{T_{0} \cup \tilde{T}}^{i}\left[\left(\left|T_{0}\right|+k+1\right) v\left(T_{0} \cup \tilde{T} \cup\{i\}\right)\right.$

$$
\begin{gathered}
\left.-(k+1) v\left(T_{0}\right)\right]+\sum_{i_{1} \in T \backslash T_{0}} \sum_{k=1}^{|N \backslash T|-1} \sum_{\substack{\tilde{T} \subseteq(N \backslash T)\{\{i\} \\
|\tilde{T}|=k}} \frac{1}{\left|T_{0}\right|+k+2} \\
\times p_{T_{0} \cup \tilde{U} \cup\left\{i_{1}\right\}}^{i}\left[\left(\left|T_{0}\right|+k+2\right) v\left(T_{0} \cup \tilde{T} \bigcup\left\{i_{1}\right\} \bigcup\{i\}\right)-v\left(T_{0}\right)\right] \\
\left(\phi_{i}(v)\right)_{T_{0}}=\frac{\left|T_{0}\right|}{\left|T_{0}\right|+1} p_{\left|T_{0}\right|} v\left(T_{0}\right)-\frac{\left(|T|-\left|T_{0}\right|\right)}{\left|T_{0}\right|+2} p_{\left|T_{0}\right|+1} v\left(T_{0}\right)
\end{gathered}
$$

That is

$$
\begin{aligned}
& +\sum_{k=1}^{|N \backslash T|-1} \frac{\left|T_{0}\right|\left(\begin{array}{c}
|N \backslash T|-1 \\
k
\end{array}\right)}{\left|T_{0}\right|+k+1} p_{\left|T_{0}\right|+k} v\left(T_{0}\right) \\
& -\sum_{k=1}^{|N \backslash T|-1} \frac{\left(|T|-\left|T_{0}\right|\right)\left(\begin{array}{c}
|N \backslash T|-1 \\
k
\end{array}\right)}{\left|T_{0}\right|+k+2} p_{\left|T_{0}\right|+k+1} v\left(T_{0}\right)
\end{aligned}
$$

$$
\begin{aligned}
& \left(\phi_{i}(v)\right)_{T_{0}}=\frac{1}{\left|T_{0}\right|+1} p_{T_{0}}^{i}\left[\left(\left|T_{0}\right|+1\right) v\left(T_{0} \bigcup\{i\}\right)-v\left(T_{0}\right)\right] \\
& +\sum_{i_{1} \in T \backslash T_{0}} \frac{1}{\left|T_{0}\right|+2} p_{T_{0} \cup\left\{i_{1}\right\}}^{i}\left[\left(\left|T_{0}\right|+2\right) v\left(T_{0} \bigcup\left\{i_{1}\right\} \bigcup\{i\}\right)-v\left(T_{0}\right)\right] \\
& \text { b) }+\sum_{k=1}^{|N| T \mid-1} \sum_{\tilde{T} \subseteq(N \mid T)\{\{i\}} \frac{1}{\left|T_{0}\right|+k+1} p_{T_{0} \cup \tilde{T} \mid=k}^{i}\left[\left(\left|T_{0}\right|+k+1\right) v\left(T_{0} \cup \tilde{T} \bigcup\{i\}\right)\right.
\end{aligned}
$$

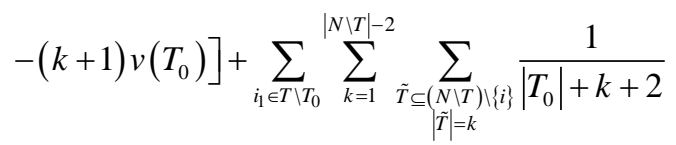

$$
\begin{aligned}
& \times p_{T_{0} \cup \tilde{T} \cup\left\{\left\{_{1}\right\}\right.}^{i}\left[\left(\left|T_{0}\right|+k+2\right) v\left(T_{0} \cup \tilde{T} \bigcup\left\{i_{1}\right\} \bigcup\{i\}\right)-v\left(T_{0}\right)\right]
\end{aligned}
$$




$$
\begin{aligned}
& \left(\phi_{i}(v)\right)_{T_{0}}=\frac{\left|T_{0}\right|}{\left|T_{0}\right|+1} p_{\left|T_{0}\right|} v\left(T_{0}\right)-\frac{1}{\left|T_{0}\right|+2} p_{\left|T_{0}\right|+1} v\left(T_{0}\right) \\
& +\sum_{k=1}^{|N \backslash T|-1} \frac{\left|T_{0}\right|\left(\begin{array}{c}
|N \backslash T|-1 \\
k
\end{array}\right)}{\left|T_{0}\right|+k+1} p_{\left|T_{0}\right|+k} v\left(T_{0}\right)-\sum_{k=1}^{|N \backslash T|-2} \frac{\left(\begin{array}{c}
|N \backslash T|-1 \\
k
\end{array}\right)}{\left|T_{0}\right|+k+2} p_{\left|T_{0}\right|+k+1} v
\end{aligned}
$$

So

Lemma 21 For $T_{0}=T\left(T_{0}\right)$ and $i \in N \backslash T$ :

$$
\begin{aligned}
&\left(\phi_{i}(v)\right)_{T_{0}}=\left[\frac{\left|T_{0}\right|}{\left|T_{0}\right|+1} p_{\left|T_{0}\right|}+\sum_{k=1}^{|N \backslash T|-2} \frac{T_{0} \mid}{\left.\mid \begin{array}{c}
|N \backslash T|-1 \\
k
\end{array}\right)} p_{\left|T_{0}\right|+k}+\frac{|N \backslash T|}{\left|T_{0}\right|} p_{|N \backslash T|-1}\right. \\
&\left.+\sum_{k=1}^{|T|-1} \frac{|N \backslash T|^{2}\left(\begin{array}{c}
|T| \\
k
\end{array}\right)}{|T|[|N \backslash T|+k]} p_{|N| T \mid+k-1}\right] v\left(T_{0}\right) \\
&\left(\phi_{i}(v)\right)_{T_{0}}=\frac{1}{\left|T_{0}\right|+1} p_{T_{0}}^{i}\left[\left(\left|T_{0}\right|+1\right) v\left(T_{0} \cup\{i\}\right)-v\left(T_{0}\right)\right] \\
& \quad+\sum_{k=1}^{|N \backslash T|-2} \sum_{\tilde{T} \subseteq(N \backslash T) \backslash\{i\}} \frac{1}{\left|T_{0}\right|+k+1} p_{T_{0} \cup \tilde{T}}^{i}\left[\left(\left|T_{0}\right|+k+1\right) v\left(T_{0} \cup \tilde{T} \cup\{i\}\right)\right.
\end{aligned}
$$

Proof.

$$
\begin{aligned}
& \left.-(k+1) v\left(T_{0}\right)\right]+\frac{1}{|N \backslash T|} p_{(N \backslash T) \backslash\{i\}}^{i}[|N \backslash T| v(N \backslash T)] \\
& +\sum_{k=1}^{|T|-1} \sum_{\tilde{\tilde{T}} \in T} \frac{1}{\mid N \backslash=k} p_{(N \backslash T) \backslash\{i\} \cup \tilde{T}}^{i}[(|N \backslash T|+k) v((N \backslash T) \cup \tilde{T})-k v(N \backslash T)] \\
& \left(\phi_{i}(v)\right)_{T_{0}}=\frac{\left|T_{0}\right|}{\left|T_{0}\right|+1} p_{\left|T_{0}\right|} v\left(T_{0}\right)+\sum_{k=1}^{|N \backslash T|-2} \frac{\left|T_{0}\right|\left(\begin{array}{c}
|N \backslash T|-1 \\
k
\end{array}\right)}{\left|T_{0}\right|+k+1} p_{\left|T_{0}\right|+k} v\left(T_{0}\right)
\end{aligned}
$$

Hence

$$
+\frac{|N \backslash T|}{|T|} p_{|N \backslash T|-1} v\left(T_{0}\right)+\sum_{k=1}^{|T|-1} \frac{|N \backslash T|^{2}\left(\begin{array}{c}
|T| \\
k
\end{array}\right)}{|T|[|N \backslash T|+k]} p_{|N \backslash T|+k-1} v\left(T_{0}\right)
$$

For the continuation, we need to introduce some notations with a view to more easily present each payoff $\phi_{i}(v)$ : for every $T_{0} \subseteq T$ or $S_{0} \varsubsetneqq N \backslash T$, we write $\left(\phi_{i}(v)\right)_{T_{0}}=\overline{\left(\phi_{i}(v)\right)_{T_{0}}} v\left(T_{0}\right)$ or $\left(\phi_{i}(v)\right)_{S_{0}}=\overline{\left(\phi_{i}(v)\right)_{S_{0}}} v\left(S_{0}\right)$ where $\overline{\left(\phi_{i}(v)\right)_{T_{0}}}$ and $\overline{\left(\phi_{i}(v)\right)_{S_{0}}}$ are given by the related lemma above. Recall that we have voluntarily forgotten probability $p_{n-1}$ in those lemmas. So we obtain:

$$
\forall i \in N \quad, \quad \phi_{i}(v)=\sum_{T_{0} \subseteq T} \overline{\left(\phi_{i}(v)\right)_{T_{0}}} v\left(T_{0}\right)+\sum_{S_{0} \cong N \backslash T} \overline{\left(\phi_{i}(v)\right)_{S_{0}}} v\left(S_{0}\right)+\frac{1}{|N|} p_{n-1} A^{v}(N)
$$

Furthermore we denote $i_{1} \in T_{0}, \quad i_{2} \in T \backslash T_{0}, \quad j_{1} \in S_{0}, \quad j_{2} \in(N \backslash T) \backslash S_{0}, \quad i_{3} \in T$ and $j_{3} \in N \backslash T$. 
Theorem 22 shows that the set of symmetric ps-values which satisfy the per-capita productivity property is not empty.

Theorem 22 Let $\phi$ be a symmetric ps-value on $N$ defined by a probability system $\left(p_{t}\right)_{t=0,1,2, \cdots, n-1}$.

$\phi$ satisfies the per-capita productivity property if and only if $\left(p_{t}\right)_{t=0,1,2, \cdots, n-2}$ is solution for the linear system defined by:

$$
\left\{\begin{array}{l}
|N \backslash T|\left|T_{0}\right| \overline{\left(\phi_{i_{1}}(v)\right)_{T_{0}}}+|N \backslash T|\left|T \backslash T_{0}\right| \overline{\left(\phi_{i_{2}}(v)\right)_{T_{0}}}-|T||N \backslash T| \overline{\left(\phi_{j_{3}}(v)\right)_{T_{0}}}=0,\left|T_{0}\right|=1,2, \cdots,|T| \\
|N \backslash T||T| \overline{\left(\phi_{i_{3}}(v)\right)_{S_{0}}}-|T|\left|S_{0}\right| \overline{\left(\phi_{j_{1}}(v)\right)_{S_{0}}}-|T|\left|(N \backslash T) \backslash S_{0}\right| \overline{\left(\phi_{j_{2}}(v)\right)_{S_{0}}}=0,\left|S_{0}\right|=1,2, \cdots, N-|T|-1
\end{array}\right.
$$

Proof. Consider a symmetric ps-value $\phi$ and a game $v \in \Gamma$ such that:

$$
\forall S \subseteq N, v(S)=v(S \cap T)+v(S \cap(N \backslash T)) \text { and } \frac{v(T)}{|T|}=\frac{v(N \backslash T)}{|N \backslash T|} \text {. Using }\left(\mathrm{E}_{1}\right)
$$

we have:

$$
\begin{aligned}
& \frac{\sum_{i \in T} \phi_{i}(v) \quad \sum_{i \in N \backslash T} \phi_{i}(v)}{|T|}-\frac{|N \backslash T|}{=}|N \backslash T| \sum_{i \in T}\left[\sum_{T_{0} \subseteq T} \overline{\left(\phi_{i}(v)\right)_{T_{0}}} v\left(T_{0}\right)+\sum_{S_{0} \varsubsetneqq N \backslash T} \overline{\left(\phi_{i}(v)\right)_{S_{0}}} v\left(S_{0}\right)\right] \\
& \\
& -|T| \sum_{i \in N \backslash T}\left[\sum_{T_{0} \subseteq T}^{\left(\phi_{i}(v)\right)_{T_{0}}} v\left(T_{0}\right)+\sum_{S_{0} \varsubsetneqq N \backslash T} \overline{\left(\phi_{i}(v)\right)_{S_{0}}} v\left(S_{0}\right)\right] \\
& =\sum_{T_{0} \subseteq T}\left[|N \backslash T| \sum \overline{\left(\phi_{i}(v)\right)_{T_{0}}}-|T| \sum_{i \in N \backslash T} \overline{\left(\phi_{i}(v)\right)_{T_{0}}}\right] v\left(T_{0}\right) \\
& +\sum_{S_{0} \varsubsetneqq N \backslash T}\left[|N \backslash T| \sum_{i \in T} \overline{\left(\phi_{i}(v)\right)_{S_{0}}}-|T| \sum_{i \in N \backslash T} \overline{\left(\phi_{i}(v)\right)_{S_{0}}}\right] v\left(S_{0}\right)
\end{aligned}
$$

Due to different lemma above, we obtain:

$$
\begin{aligned}
& \frac{\sum_{i \in T} \phi_{i}(v)}{|T|}-\frac{\sum_{i \in N \backslash T} \phi_{i}(v)}{|N \backslash T|} \\
& =\sum_{T_{0} \subseteq T}\left[|N \backslash T|\left(\sum_{i \in T_{0}}\left(\overline{\left.\phi_{i}(v)\right)_{T_{0}}}+\sum_{i \in T \backslash T_{0}} \overline{\left(\phi_{i}(v)\right)_{T_{0}}}\right)-|T| \sum_{i \in N \backslash T} \overline{\left(\phi_{i}(v)\right)_{T_{0}}}\right] v\left(T_{0}\right)\right. \\
& +\sum_{S_{0} \varsubsetneqq N \backslash T}\left[|N \backslash T| \sum_{i \in T} \overline{\left(\phi_{i}(v)\right)_{S_{0}}}-|T|\left(\sum_{i \in S_{0}}^{\left(\phi_{i}(v)\right)_{S_{0}}}+\sum_{i \in(N \backslash T) \backslash S_{0}} \overline{\left(\phi_{i}(v)\right)_{S_{0}}}\right)\right] v\left(S_{0}\right)
\end{aligned}
$$

That is

$$
\begin{aligned}
& \frac{\sum_{i \in T} \phi_{i}(v)}{|T|}-\frac{\sum_{i \in N \backslash T} \phi_{i}(v)}{|N \backslash T|} \\
& =\sum_{T_{0} \subseteq T}\left[|N \backslash T|\left(\left|T_{0}\right| \overline{\left(\phi_{i_{1}}(v)\right)_{T_{0}}}+\left|T \backslash T_{0}\right| \overline{\left(\phi_{i_{2}}(v)\right)_{T_{0}}}\right)-|T||N \backslash T| \overline{\left(\phi_{j_{3}}(v)\right)_{T_{0}}}\right] v\left(T_{0}\right) \\
& +\sum_{S_{0} \varsubsetneqq N \backslash T}\left[|N \backslash T||T| \overline{\left(\phi_{i_{3}}(v)\right)_{S_{0}}}-|T|\left(\left|S_{0}\right| \overline{\left(\phi_{j_{1}}(v)\right)_{S_{0}}}+\left|(N \backslash T) \backslash S_{0}\right| \overline{\left(\phi_{j_{2}}(v)\right)_{S_{0}}}\right)\right] v\left(S_{0}\right)
\end{aligned}
$$


Consider the following linear system:

$\left(\mathrm{E}_{2}\right)$ :

$$
\left\{\begin{array}{l}
|N \backslash T|\left(\left|T_{0}\right| \overline{\left(\phi_{i_{1}}(v)\right)_{T_{0}}}+\left|T \backslash T_{0}\right| \overline{\left(\phi_{i_{2}}(v)\right)_{T_{0}}}\right)-|T||N \backslash T| \overline{\left(\phi_{j_{3}}(v)\right)_{T_{0}}}=0, T_{0} \subseteq T \\
|N \backslash T||T| \overline{\left(\phi_{i_{3}}(v)\right)_{S_{0}}}-|T|\left(\left|S_{0}\right| \overline{\left(\phi_{j_{1}}(v)\right)_{S_{0}}}+\left|(N \backslash T) \backslash S_{0}\right| \overline{\left(\phi_{j_{2}}(v)\right)_{S_{0}}}\right)=0, S_{0} \varsubsetneqq N \backslash T
\end{array}\right.
$$

Once more using different lemma above, on can easily notice that for each $T_{0} \subseteq T$ and $S_{0} \varsubsetneqq N \backslash T, \overline{\left(\phi_{i}(v)\right)_{T_{0}}}$ and $\overline{\left(\phi_{i}(v)\right)_{S_{0}}}$ just depend on the size of $T_{0}$ and $S_{0}$. Consequently $\left(\mathrm{E}_{2}\right)$ is equivalent to $\left(\mathrm{E}_{3}\right)$ where:

$\left(\mathrm{E}_{3}\right)$ :

$$
\left\{\begin{array}{l}
|N \backslash T|\left|T_{0}\right| \overline{\left(\phi_{i_{1}}(v)\right)_{T_{0}}}+|N \backslash T|\left|T \backslash T_{0}\right| \overline{\left(\phi_{i_{2}}(v)\right)_{T_{0}}}-|T||N \backslash T| \overline{\left(\phi_{j_{3}}(v)\right)_{T_{0}}}=0,\left|T_{0}\right|=1,2, \cdots,|T| \\
|N \backslash T||T| \overline{\left(\phi_{i_{3}}(v)\right)_{S_{0}}}-|T|\left|S_{0}\right| \overline{\left(\phi_{j_{1}}(v)\right)_{S_{0}}}-|T|\left|(N \backslash T) \backslash S_{0}\right| \overline{\left(\phi_{j_{2}}(v)\right)_{S_{0}}}=0,\left|S_{0}\right|=1,2, \cdots, N-|T|-1
\end{array}\right.
$$

It is straightforward that $p_{0}=p_{1}=\cdots=p_{n-2}=0$ is solution for $\left(\mathrm{E}_{3}\right)$. Hence for $p_{n-1}=1, \phi$ satisfies the per-capita productivity property. More generally, every solution $\left(p_{0}, p_{1}, p_{2}, \cdots, p_{n-2}\right)$ for $\left(\mathrm{E}_{3}\right)$ such that $\forall t=0,1,2, \cdots, n-2$, $p_{t} \geq 0$ and $\sum_{t=0}^{n-2} p_{t} \leq 1$ induces a symmetric ps-value which exhibits the per-capita productivity property, since we just have to take $p_{n-1}=1-\sum_{t=0}^{n-2} p_{t}$.

Conversely, consider a probability system $\left(p_{t}\right)_{t=0,1,2, \cdots, n-1}$ and assume that there exists $T_{0} \subseteq T$ such that:

$$
|N \backslash T|\left|T_{0}\right| \overline{\left(\phi_{i_{1}}(v)\right)_{T_{0}}}+|N \backslash T|\left|T \backslash T_{0}\right| \overline{\left(\phi_{i_{2}}(v)\right)_{T_{0}}}-|T||N \backslash T| \overline{\left(\phi_{j_{3}}(v)\right)_{T_{0}}} \neq 0 \text {. We de- }
$$
fine the game $v \in \Gamma$ as follows:

$$
v(T)=\frac{|T|}{|N \backslash T|} v(N \backslash T) \text { and } v(S)=\left\{\begin{array}{l}
v\left(T_{0}\right) \neq 0, S=T_{0} \\
0, S \in 2^{T} \backslash T_{0} \text { or } S \subseteq N \backslash T \\
v(S \cap T)+v(S \cap(N \backslash T)) \text { else }
\end{array}\right.
$$

That implies:

$$
\begin{aligned}
& \frac{\sum_{i \in T} \varphi_{i}(v)}{|T|}-\frac{\sum_{i \in N \backslash T} \varphi_{i}(v)}{|N \backslash T|} \\
& =\left[|N \backslash T|\left|T_{0}\right| \overline{\left(\phi_{i_{1}}(v)\right)_{T_{0}}}+|N \backslash T|\left|T \backslash T_{0}\right| \overline{\left(\phi_{i_{2}}(v)\right)_{T_{0}}}-|T||N \backslash T| \overline{\left(\phi_{j_{3}}(v)\right)_{T_{0}}}\right] v\left(T_{0}\right) \\
& \neq 0
\end{aligned}
$$

That concludes the proof.

Example 1 With notations above, let us fix $N=\{1,2,3\}$ and $v(12)=v(1)+v(2), v(13)=v(1)+v(3), v(1)=\frac{v(23)}{2}$ that is $T=\{1\}$.

Player 1 payoff: Using lemma 16, we have 


$$
\left(\phi_{1}(v)\right)_{\{1\}}=\left[p_{0}+\sum_{k=1}^{1} \frac{\left(\begin{array}{l}
2 \\
k
\end{array}\right)}{1+k} p_{k}\right] v(\{1\})=\left[p_{0}+p_{1}\right] v(\{1\}) .
$$

Lemma 17 gives $\left(\phi_{1}(v)\right)_{\{2\}}=\left[\frac{1}{2} p_{1}\right] v(\{2\})$ and $\left(\phi_{1}(v)\right)_{\{3\}}=\left[\frac{1}{2} p_{1}\right] v(\{3\})$.

Thus

$$
\phi_{1}(v)=\left[p_{0}+\sum_{k=1}^{1} \frac{\left(\begin{array}{l}
2 \\
k
\end{array}\right)}{1+k} p_{k}\right] v(\{1\})+\left[\frac{1}{2} p_{1}\right] v(\{2\})+\left[\frac{1}{2} p_{1}\right] v(\{3\})+\frac{1}{3} p_{2} A^{v}(N)
$$

Player 2 payoff:

By lemma 20, we have $\left(\phi_{2}(v)\right)_{\{1\}}=\left[\frac{1}{2} p_{1}+2 p_{1}\right] v(\{1\})=\left[\frac{5}{2} p_{1}\right] v(\{1\})$.

And lemma 19 implies that

$$
\left(\phi_{2}(v)\right)_{\{2\}}=\left[p_{0}-\frac{1}{2} p_{1}+\frac{\left(\begin{array}{l}
1 \\
1
\end{array}\right)}{2} p_{1}\right] v(\{2\})=\left[p_{0}\right] v(\{2\}) \text {. }
$$

Due to lemma 18, we have $\left(\phi_{2}(v)\right)_{\{3\}}=\left[-\frac{1}{2} p_{1}\right] v(\{3\})$.

Hence:

$$
\begin{aligned}
& \phi_{1}(v)= {\left[p_{0}+p_{1}\right] v(\{1\})+\left[\frac{1}{2} p_{1}\right] v(\{2\})+\left[\frac{1}{2} p_{1}\right] v(\{3\})+\frac{1}{3} p_{2} A^{v}(N) . } \\
& \phi_{2}(v)= {\left[\frac{5}{2} p_{1}\right] v(\{1\})+\left[p_{0}\right] v(\{2\})+\left[-\frac{1}{2} p_{1}\right] v(\{3\})+\frac{1}{3} p_{2} A^{v}(N) . } \\
& \phi_{3}(v)=\left[\frac{5}{2} p_{1}\right] v(\{1\})+\left[-\frac{1}{2} p_{1}\right] v(\{2\})+\left[-p_{0}\right] v(\{3\})+\frac{1}{3} p_{2} A^{v}(N) . \\
& 2 \phi_{1}(v)-\left[\phi_{2}(v)+\phi_{3}(v)\right]
\end{aligned}
$$

It is clear that $=\left[2 p_{0}-3 p_{1}\right] v(\{1\})+\left[-p_{0}+\frac{3}{2} p_{1}\right] v(\{2\})+\left[-p_{0}+\frac{3}{2} p_{1}\right] v(\{3\})$

That leads to the linear system:

$$
\left\{\begin{array}{l}
2 p_{0}-3 p_{1}=0 \\
-p_{0}+\frac{3}{2} p_{1}=0
\end{array}\right.
$$

It comes out that each system $\left(p_{0}, p_{1}\right)$ such that $-p_{0}+\frac{3}{2} p_{1}=0,0 \leq p_{0}$, $0 \leq p_{1}, \quad p_{0}+p_{1} \leq 1$ exhibits a ps-value (with $p_{2}=1-\left[p_{0}+p_{1}\right]$ ) satisfying the per-capita productivity property.

Considering the game $v=u_{\{1\}}+2 u_{\{2,3\}}$ : 
For $p_{0}=\frac{3}{10}, \quad p_{1}=\frac{2}{10}, \quad p_{2}=\frac{5}{10}$, we obtain $\phi_{1}(v)=\phi_{2}(v)=\phi_{3}(v)=\frac{4}{3}$ and $\sum_{i \in N} \phi_{i}(v)=4>v(N)$.

When $p_{0}=\frac{3}{5}, \quad p_{1}=\frac{2}{5}, \quad p_{2}=0$, we obtain $\phi_{1}(v)=\phi_{2}(v)=\phi_{3}(v)=1$ and $\sum_{i \in N} \phi_{i}(v)=3=v(N)$.

In a society $N$, it is clear that one can imagine various ways to express solidarity among players. Whatever policy is put in place, we would like the resulting sharing meets the intuitive rational behavior. One can underline two variants in evaluating a value which expresses some solidarity: its solidary social behavior and its configuration of the society $N$ into coalitions. For the Solidarity value of Nowak and Radzik (1994) the solidary social behavior is the evenly sharing of the average marginal contribution of every formed coalition, and all coalitions are likely to be formed with the same probability. However a social behavior could not be suitable for any configuration of $N$ in any game $v$. In other words, one can understand that the problem in some non intuitive payoffs under the Solidarity value could find a solution in ps-values, as they allow a re-configuration of the society $N$ by different probability systems. This way example 1 , shows that for $p_{0}=\frac{3}{5}, p_{1}=\frac{2}{5}$ and $p_{2}=0$, we obtain for $v=u_{\{1\}}+2 u_{\{2,3\}}$ a solution which maintaining the social behavior implemented in the Solidarity value, satisfies the per-capita productivity property by a re-configuration of the society $N$ since it is not allowed the formation of the grand coalition with $p_{2}=0$.

\section{Concluding Comments}

To understand how collectively produced income is individually shared is a tough challenge, since the game only tells the capacity of production of every coalition. The challenge persists, even when Shapley assumes for simplicity that all players end up by cooperating as members of the grand coalition $N$, and only need to share its production $v(N)$. He assumes equal chance of joining $N$ at any given step of its constitution. Weber's work on probabilistic values shows that the information on how each player is likely to be picked at each stage en route to building $N$ plays a key role. Fair sharing formulae have been provided by Shapley, and later generalized by Weber who also characterized the Shapley Value. Our contribution on this paper investigates on how the Shapley and Weber's combined setting works when Radzik and Nowak's solidarity concern is implemented. Our formulae for the transposed setting have been obtained, and they disclose that the Radzik-Nowak solidarity value is to the probabilistic solidarity value what the Shapley value is to the probabilistic value.

It is worth remarking that many social environments for production do not comply with Weber and Shapley's assumption that a player joins a coalition at random (be it with equality of chance or following a priori setting of probability 
distribution). For example in Aumann and Dreze (1974), Owen et al. (1977) and Hart and Kurz (1983), the idea of coalition structures for players prevails following the argument that a group of players may find it to their advantage to join forces in some situations, and to act separately in others, depending on the opportunities that they may find with players outside the group. In Hellman and Peretz (2018), players are located within a priori graph structure that limits coalition formation. In many real-life contexts, a player's individual choice matters and gives him individual but partial influence to the picking of whom to admit at a given stage into a coalition being built. So, the struggle for individual interest in player interaction causes endogenous coalition formation issues, and must result to serious impacts in individual shares if we assume that the grand coalition $N$ is attained, all players being rational. We may assume that all impacts of players' interest on the sharing outcome originate from the game itself with no exogenous input and must be accounted for by processing the data that are found in the function $v$ that defines that game. Therefore, the probability of a player being admitted is impacted endogenously by the rational behavior of players. So for further work, it is naturally appealing to examine what becomes the concept of ps-values when the probability system is a consequence of players' interest in the game.

\section{Conflicts of Interest}

The authors declare no conflicts of interest regarding the publication of this paper.

\section{References}

Aumann, R. J., \& Dreze, J. H. (1974). Cooperative Games with Coalition Structures. International Journal of Game Theory, 3, 217-237. https://doi.org/10.1007/BF01766876

Casajus, A., \& Huettner, F. (2014). On a Class of Solidarity Values. European Journal of Operational Research, 236, 583-591. https://doi.org/10.1016/j.ejor.2013.12.015

Hart, S., \& Kurz, M. (1983). Endogenous Formation of Coalitions. Econometrica: Journal of the Econometric Society, 51, 1047-1064. https://doi.org/10.2307/1912051

Hellman, Z., \& Peretz, R. (2018). Values for Cooperative Games over Graphs and Games with Inadmissible Coalitions. Games and Economic Behavior, 108, 22-36. https://doi.org/10.1016/j.geb.2016.12.007

Nowak, A. S., \& Radzik, T. (1994). A Solidarity Value Forn-Person Transferable Utility Games. International Journal of Game Theory, 23, 43-48. https://doi.org/10.1007/BF01242845

Owen, G., Henn, R., \& Moeschlin, O. (1977). Essays in Mathematical Economics and Game Theory. In R. Henn, \& O. Moeschlin (Eds.), Lecture Notes in Economics and Mathematical Systems. New York: Springer.

Shapley, L. S. (1953). A Value for n-Person Games. Contributions to the Theory of Games, 2, 307-317. https://doi.org/10.1515/9781400881970-018

Weber, R. J. (1988). Probabilistic Values for Games. In E. R. Alvin (Ed.), The Shapley Value. Essays in Honor of Lloyd S. Shapley (pp. 101-120). Cambridge: Cambridge University Press. https://doi.org/10.1017/CBO9780511528446.008 